\title{
Mapping Iso-Orientation Columns by Contrast Agent- Enhanced Functional Magnetic Resonance Imaging: Reproducibility, Specificity, and Evaluation by Optical Imaging of Intrinsic Signal
}

\author{
Mitsuhiro Fukuda, ${ }^{\star}$ Chan-Hong Moon, ${ }^{\star}$ Ping Wang, and Seong-Gi Kim \\ Departments of Radiology and Neurobiology, University of Pittsburgh, Pittsburgh, Pennsylvania 15203
}

\begin{abstract}
Activation resembling ocular dominance or orientation columns has been mapped with high-resolution functional magnetic resonance imaging (fMRI). However, the neuronal interpretation of these functional maps is unclear because of the poor sensitivity of fMRI, unknown point spread function (PSF), and lack of comparison with independent techniques. Here we show that cerebral blood volume (CBV)-weighted fMRI with a blood plasma contrast agent (monocrystalline iron oxide nanoparticles), in combination with continuous temporally encoded stimulation, can map columnar neuronal activity in the cat primary visual cortex with high sensitivity, selectivity, and reproducibility. We examined hemodynamic response PSF by comparing these CBV-based signals with oxygen metabolism-based negative blood oxygenation level-dependent signals. A significant positive correlation exists between CBV- and metabolism-based iso-orientation maps, suggesting that the hemodynamic PSF is narrower than intercolumn distances. We also compared CBV-based fMRI with optical intrinsic signal (OIS) imaging, a technique that identifies sites of increased neuronal activity, to investigate neuronal correlation. Iso-orientation maps obtained by fMRI and OIS were well matched, indicating that areas of the highest orientation-selective CBV signals correspond to sites of increased neural activity. Using CBV-based fMRI, we successfully mapped orientation-selective functional architecture in the medial bank of the visual cortex, an area inaccessible to OIS imaging. Thus, we conclude that contrast agent-based fMRI, in combination with continuous temporally encoded stimulation, is a highly sensitive technique capable of mapping neural activity at the resolution of functional columns without depth limitation.
\end{abstract}

Key words: BOLD; CBF; hemodynamic response; capillary; visual cortex; cerebral blood flow

\section{Introduction}

Activation patterns resembling ocular dominance and orientation columns have been demonstrated with high-resolution functional magnetic resonance imaging ( $\mathrm{fMRI}$ ) in human and cat primary visual cortex in response to monocular eye stimulation (Menon et al., 1997; Menon and Goodyear, 1999; Dechent and Frahm, 2000; Cheng et al., 2001; Goodyear and Menon, 2001) and orientation-selective stimulation (Kim et al., 2000; Duong et al., 2001; Zhao et al., 2005). However, the utility of highresolution $\mathrm{fMRI}$ is still problematic because of its poor sensitivity, unknown point spread function (PSF), and a lack of confirmation by independent techniques.

Received July 20, 2006; revised 0ct. 5, 2006; accepted 0ct. 8, 2006.

This work was supported by National Institutes of Health (NIH) Grants NS44589, EB003324, and EB003375 and the McKnight Foundation. The 9.4 T MRI system was funded in part by NIH Grant RR17239. We thank Sung-Hong Park for development of MR venography, Michelle Tasker for animal preparation, Dr. Amiram Grinvald for encouraging fMRI versus optical imaging experiments, and Dr. Dae-Shik Kim for insightful comments on a previous version of this manuscript. We are also grateful to Kristy Hendrich for critical reading and editing of our manuscript.

${ }^{*}$ M.F. and C.-H.M. contributed equally to this work.

Correspondence should be addressed to Dr. Seong-Gi Kim, Departments of Radiology and Neurobiology, University of Pittsburgh, 3025 East Carson Street, Pittsburgh, PA 15203. E-mail: kimsg@pitt.edu.

DOI:10.1523/JNEUROSCI.3098-06.2006

Copyright $\odot 2006$ Society for Neuroscience $\quad$ 0270-6474/06/2611821-12\$15.00/0
Although an increase in fMRI signal is closely correlated with an increase in neuronal activity on a large supramillimeter scale (Heeger et al., 2000; Ress et al., 2000; Logothetis et al., 2001), this finding may not be applicable on a submillimeter columnar scale. When a single cortical column is activated (Kennerley et al., 2005), the highest fMRI signal will appear at the site of increased neuronal activity (referred to as an "active column") regardless of the PSF (see Fig. 1 A). However, if multiple columns are activated, successfully determining the location of active columns within fMRI maps will be dictated by the PSF width relative to the distance between neighboring active columns (see Fig. $1 B$ ). Because of this limitation, it is conceivable that the highest signals induced by bars of $0^{\circ}\left(90^{\circ}\right)$ orientation may appear at the location of $90^{\circ}$ $\left(0^{\circ}\right)$ orientation columns. Therefore, it is absolutely imperative to confirm our interpretation of submillimeter-resolution fMRI columnar maps by comparing them with conventional techniques such as optical intrinsic signal (OIS) imaging, 2-deoxyglucose (2-DG) imaging, or multiple-unit recording. Of these, OIS imaging is the best choice. OIS maps can serve as a reference to a spatial pattern of neuronal activity; a good correlation between spiking activity and OIS has already been observed (Grinvald et al., 1986; Maldonado et al., 1997; Bosking et al., 2002) (see also Blasdel and Salama, 1986). In contrast, the 2-DG technique cannot be per- 
Table 1. Data analysis parameters

\begin{tabular}{|c|c|c|c|c|c|}
\hline Experiments & $\begin{array}{l}\text { Reproducibility of } \\
\text { CBV-weighted } \\
\text { fMRI }\end{array}$ & $\begin{array}{l}\text { Spatial frequency } \\
\text { dependency of } \\
\text { CBV-weighted fMRI }\end{array}$ & $\begin{array}{l}\mathrm{CMRO}_{2} \text {-based fMRI } \\
\text { versus CBV-weighted } \\
\text { fMRI }\end{array}$ & $\begin{array}{l}\text { CBV-weighted fMRI versus } 570 \mathrm{~nm} \\
\text { OIS }\end{array}$ & $\begin{array}{l}\text { CBV-weighted fMRI } \\
\text { in medial bank }\end{array}$ \\
\hline Number of animals & 5 & 5 & 4 & 6 & 2 \\
\hline $\begin{array}{l}\text { Number of repetition for the same orientation } \\
\text { per scan } \times \text { number of scans }\end{array}$ & $10 \times 1$ & $10 \times 1$ & $\begin{array}{c}10 \times 3-5\left(\mathrm{CMRO}_{2}\right) \\
10 \times 5(\mathrm{CBV})\end{array}$ & $10 \times 2-5(\mathrm{fMRI}), 10 \times 2-8(\mathrm{OIS})$ & $10 \times 2$ \\
\hline Spatial low-pass filter for EPI image ${ }^{a}$ & $1 /(0.31 \mathrm{~mm})$ & $1 /(0.31 \mathrm{~mm})$ & $1 /(0.31 \mathrm{~mm})$ & $1 /(0.31 \mathrm{~mm})$ & $\begin{array}{l}1 /(0.28 \mathrm{~mm})^{e} \\
1 /(0.31 \mathrm{~mm})\end{array}$ \\
\hline Spatial high-pass filter for iso-orientation map ${ }^{a}$ & None & None & $1 /(4.9 \mathrm{~mm})$ & $1 /(4.9 \mathrm{~mm})$ & None \\
\hline Spatial low-pass filter for iso-orientation map ${ }^{\text {a }}$ & None & None & $1 /(0.3 \mathrm{~mm})$ & None & None \\
\hline Hemodynamic response time (s) & 13 & 13 & $\begin{array}{l}16-18\left(\mathrm{CMRO}_{2}\right)^{b}, 10^{c} \\
13(\mathrm{CBV})\end{array}$ & $10^{d}, 13$ & 13 \\
\hline $\begin{array}{l}\text { Number of pixels or area within ROl for quanti- } \\
\text { tative comparison }\end{array}$ & 20,301 & $2.1 \times 2.1 \mathrm{~mm}^{2}$ & $11,774-21,828$ & $(1.21-2.38) \times(3.79-5.72) \mathrm{mm}^{2}$ & 20,301 \\
\hline
\end{tabular}

${ }^{a}$ Cutoff frequency is given at full-width half-maximum.

${ }^{b}$ For more accurate assignment of stimulation orientation to the $\mathrm{CMRO}_{2}$-related $\mathrm{fMRI}$ maps, the response time-to-peak was determined for each cat after fitting the signal time course to the sinusoidal curve.

COne CBV-weighted fMRI measurement was performed with the 10 s intermittent stimulation paradigm 1 week before studies of $\mathrm{CMRO}_{2}$-based fMRI. Imaging areas from different days were coregistered based on vessel anatomical images. Hemodynamic response time was $10 \mathrm{~s}$.

${ }^{d}$ In one cat, the $10 \mathrm{~s}$ intermittent stimulation paradigm was used for both fMRI and OIS imaging. Hemodynamic response time was $10 \mathrm{~s}$ in both measurements.

${ }^{e} \mathrm{FOV}$ is $1.8 \times 1.8 \mathrm{~mm}^{2}$

formed with multiple stimulus configurations, and multiple-unit recording lacks sufficient field of view because of the point-bypoint measurement.

In the present studies, we performed high-resolution fMRI at the resolution of orientation columns in the visual area of isoflurane-anesthetized cats. To achieve high specificity and sensitivity for orientation column mapping, we adopted two techniques: the injection of a blood plasma contrast agent [monocrystalline iron oxide nanoparticles $(\mathrm{MION})]$ for cerebral blood volume (CBV) weighting (Kennan et al., 1998; Mandeville et al., 1998, 2004; van Bruggen et al., 1998; Zhao et al., 2006) and a paradigm of temporal encoding in conjunction with continuous stimulation (Engel et al., 1994; Sereno et al., 1995; Kalatsky and Stryker, 2003). Three major issues were examined: (1) reproducibility and spatial specificity of the orientation-selective fMRI signals, (2) PSF of the hemodynamic fMRI signal, which was investigated by comparing iso-orientation maps obtained from CBV-weighted fMRI with cerebral metabolic rate of oxygen $\left(\mathrm{CMRO}_{2}\right)$-based fMRI maps, and (3) the neural interpretation of iso-orientation fMRI maps, which was tested by correlation with OIS maps.

\section{Materials and Methods}

Animal preparation. With approval of the Institutional Animal Care and Use Committee at the University of Pittsburgh, a total of 13 cats (1.2-2.3 $\mathrm{kg}, 9-23$ weeks old) was studied by fMRI; each cat was used for multiple experiments (Table 1), and six of these cats were also studied by optical imaging for comparison. Each cat was initially treated with atropine sulfate $(0.05 \mathrm{mg} / \mathrm{kg}, \mathrm{i} . \mathrm{m}$.). Anesthesia was induced by a mixture of ketamine $(10-20 \mathrm{mg} / \mathrm{kg}, \mathrm{i} . \mathrm{m}$.) and xylazine ( $1 \mathrm{mg} / \mathrm{kg}$, i.m.); a mixture of air and oxygen maintaining $30-35 \% \mathrm{O}_{2}$ was used with $2 \%$ isoflurane during surgery and with either $0.8-1.2 \%$ isoflurane $(n=11)$ or $\alpha$-chloralose ( $n=2 ; 30 \mathrm{mg} / \mathrm{kg}$, i.v.; maintenance, $5 \mathrm{mg} / \mathrm{kg}$, i.v.) during fMRI and OIS studies, except for the comparison study between the $\mathrm{CMRO}_{2}$-based fMRI and CBV-weighted fMRI. In this experiment, a mixture of $30-35 \%$ $\mathrm{O}_{2}$ and $65-70 \% \mathrm{~N}_{2} \mathrm{O}$ was used with $0.8-1.2 \%$ isoflurane. The cat was paralyzed with pancuronium bromide $\left(0.2 \mathrm{mg} \cdot \mathrm{kg}^{-1} \cdot \mathrm{hr}^{-1}\right.$, i.v. $)$, and appropriate contact lenses were used. For MRI studies, the cat was placed in a cradle and secured in a normal postural position by a customdesigned head frame. The cranium was exposed for placement of a surface coil. After completion of MRI experiments, the inner contour of the coil was traced on the cranium as a spatial reference before removal. The cat was then moved to the room adjacent to the MR system for the purpose of optical imaging and was secured in a stereotaxic apparatus. A cranial window was made over the marked region, the dura was incised and reflected, and the exposed cortical surface was covered by agarose ( $\sim 1 \%$ in saline). Throughout the experiments, rectal temperature, endtidal $\mathrm{CO}_{2}$, and mean arterial blood pressure were maintained within a normal physiological range $\left[38.1 \pm 0.2^{\circ} \mathrm{C}, 3.5 \pm 0.1 \%\right.$, and $99.6 \pm 12.1$ $\operatorname{mmHg}(n=13)$, respectively].

Visual stimulation. Visual responses were induced by binocularly presenting $100 \%$ contrast square-wave gratings, which were generated using either software from Cogent Graphics (John Romaya, Wellcome Department of Imaging Neuroscience, London, UK; http://www.vislab.ucl. ac.uk/Cogent/index.html) or VSG2/5 (Cambridge Research Systems, Kent, UK). Spatial frequency of the gratings was $0.1 \mathrm{cycle} /{ }^{\circ}\left(0.3 \mathrm{cycle} /{ }^{\circ}\right.$ was used in comparison studies when noted). The gratings drifted at 2 cycles/s, with the direction of motion reversed every $0.5 \mathrm{~s}$. Stimulation was synchronized with image acquisition in fMRI and OIS experiments. An identical visual stimulation paradigm was used in both fMRI and OIS measurements. The $80 \mathrm{~s}$ cyclical continuous stimulation paradigm was used in most studies, during which eight orientations were presented $\left[0^{\circ}\right.$ (horizontal) to $157.5^{\circ}, 22.5^{\circ}$ increments, $10 \mathrm{~s} \mathrm{each}$ ] without gaps between stimuli. In two cats (Table 1), the $80 \mathrm{~s}$ cyclical intermittent stimulation paradigm (similar to block-design stimulation presentation) was additionally used, in which four orientations [0 (horizontal), 45, 90, and $135^{\circ}$, $10 \mathrm{~s}$ each] were alternated with $10 \mathrm{~s}$ homogeneous gray. In both stimulation paradigms, the duration of one full stimulation cycle was $80 \mathrm{~s}$, and it was repeated 10 times for a total presentation time of $800 \mathrm{~s}$ (i.e., one scan).

MRI. All MRI experiments were performed on a 9.4 tesla horizontal magnet with a clear boar size of $31 \mathrm{~cm}$ (Varian, Palo Alto, CA) using a surface radio frequency $(\mathrm{RF})$ coil [inner diameter, $2.5 \mathrm{~cm}(n=9)$ or 1.6 $\mathrm{cm}(n=4)]$ placed over the primary visual cortex. The position of the functional imaging slice was determined based on a flow-compensated, gradient-recalled, three-dimensional (3-D) venographic image [repetition time (TR), $50 \mathrm{~ms}$; echo time (TE), $20 \mathrm{~ms}$; data matrix, $512 \times 256 \times$ 256; field of view (FOV), $\sim 4.0 \times 2.0 \times 2.0 \mathrm{~cm}$ (dependent on brain size); isotropic resolution, $\sim 78 \mu \mathrm{m}$ ) (Park and Kim, 2005). Based on vascular patterns and gyral curvatures from the 3 -D venogram, a location of the 1 -mm-thick fMRI slice was chosen in the visual cortex in which vessels were perpendicular to the slice. For direct comparison, a twodimensional (2-D) gradient-recalled anatomic image was also acquired (TR, 50 ms; TE, 15-20 ms; data matrix, $256 \times 256$; a typical FOV, $2 \times 2$ $\mathrm{cm}$ ) with the same FOV, slice thickness, and position as fMRI to avoid a potential spatial misregistration resulting from the use of an anatomical image reconstructed from 3-D venogram.

For CBV-weighted fMRI, a bolus of dextran-coated MION contrast 

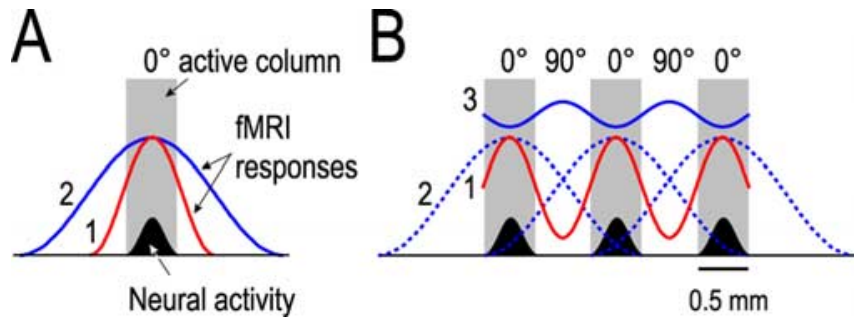

Figure 1. Schematics for spatial profiles of fMRI signals across columns. $\boldsymbol{A}$, Single-column activation with two hypothetical PSFs (1, narrow; 2 , broad). $\boldsymbol{B}$, Multiple-column activation with the same PSFs shown in $\boldsymbol{A}$. Assuming that the hemodynamic response induced by simultaneous stimulation of multiple columns is a linear summation of responses induced by individual column stimulation, solid blue trace (3) is the hypothetical response to a broad PSF (2). Note that, although individual $\mathrm{fMRI}$ responses are centered at $0^{\circ}$ active columns, highest fMRI signal changes appear at the location of the $90^{\circ}$ active column in profile 3 .

agent (typically $10 \mathrm{mg} \mathrm{Fe} / \mathrm{kg}$ body weight; obtained from the laboratory of Dr. Ralph Weissleder, Massachusetts General Hospital, Boston, MA) was injected into either the femoral or cephalic vein. The intravascular injection of MION contrast agent enhances the sensitivity of fMRI in microvessels and induces a high susceptibility effect in large vessels, which reduces large vessel contributions (Kennan et al., 1998; Mandeville et al., 1998; van Bruggen et al., 1998). In our previous studies with similar imaging parameters at $9.4 \mathrm{~T}$, the use of $10 \mathrm{mg}$ Fe $/ \mathrm{kg}$ MION improved the fMRI sensitivity in the cat visual cortex (Zhao et al., 2006). MION has a long intravascular half-life and distributes throughout the blood plasma in the cortical vessels; fMRI signal with iron oxide is mainly weighted by changes in plasma CBV in microvessels (Kennan et al., 1998; Mandeville et al., 1998; van Bruggen et al., 1998). $\mathrm{CMRO}_{2}$-based fMRI (without contrast agent) was obtained by reducing stimulus-induced cerebral blood flow $(\mathrm{CBF})$ and $\mathrm{CBV}$ with the aid of intravenous infusion of a vasodilator, sodium nitroprusside (sNP) $(0.36-2.81 \mathrm{mg} / \mathrm{kg}$ for one scan) (Fukuda et al., 2006; Nagaoka et al., 2006). All functional images were obtained during 800 -s-long visual stimulation using a gradient-echo four-shot echo planar imaging (EPI) sequence; typical imaging parameters were as follows: FOV, $2 \times 2 \mathrm{~cm}$; data matrix, $128 \times 128$ (i.e., in-plane resolution of $156 \times 156 \mu \mathrm{m} /$ pixel), slice thickness, $1 \mathrm{~mm}$; TR, $2 \mathrm{~s}$; TE, 10 ms for CBV-weighted fMRI. The $\mathrm{CMRO}_{2}$-based fMRI columnar mapping was performed on four cats before MION injection with exactly the same imaging parameters used for the CBV-weighted fMRI studies, except TE was $18 \mathrm{~ms}$.

Optical imaging. After the fMRI studies, OIS imaging was performed on a custom-built imaging system (Moon et al., 2004). The camera (FOV, $1.9 \times 1.4 \mathrm{~cm} ; 29 \times 29 \mu \mathrm{m} /$ pixel) was positioned above the MR imaging area so that the pial vessel pattern closely matched that of the MR image. The focus plane was $0-800 \mu \mathrm{m}$ below the cortical surface. The wavelength used was $570 \pm 10 \mathrm{~nm}$ [an isosbestic point of oxyhemoglobin and deoxyhemoglobin $(\mathrm{dHb})$ ]. Images were acquired with temporal resolution of $1 / 30 \mathrm{~s}$.

Data analysis. All data analysis was performed using custom-made programs with visual C ++ (Microsoft, Seattle, WA) and Matlab (MathWorks, Natick, MA). Differences attributable to anesthetics, stimulation paradigm, RF coil, and optical imaging focus plane essentially could not be observed in the results. Thus, we pooled all data together.

Image interpolation. To obtain a spatial resolution of fMRI similar to OIS maps, all of the fMRI data were interpolated by zero filling in $k$-space from $128 \times 128$ matrix to $512 \times 512$ matrix, resulting in a typical nominal in-plane resolution of $39 \times 39 \mu \mathrm{m} /$ pixel. Before zero filling, the high-frequency components in $k$-space data were apodized to avoid the ringing artifact that resulted from subsequent zero filling. These procedures did not change the activation patterns (see Fig. $4 E$ ). To match the temporal resolution of OIS to fMRI data, we averaged each of 60 consecutive OIS frame images during $800 \mathrm{~s}$ recording, resulting in a temporal resolution of 2 s/image.

Generation of temporally encoded iso-orientation maps. To generate temporally encoded maps, Fourier analysis was applied to signals contin- uously recorded for $800 \mathrm{~s}$ (10 stimulation cycles) on a pixel-by-pixel basis (Engel et al., 1997; Kalatsky and Stryker, 2003). The signal was modeled using sinusoidal functions with multiple-frequency components, with magnitude and phase described by

$$
S(x, y, t)=\sum_{i=0}^{\infty} a_{i}(x, y) \cos \left\{2 \pi f_{i} t+\varphi_{i}(x, y)\right\},
$$

where $S(x, y, t)$ is the signal intensity at position $x$ and $y$ at time $t, a_{i}(x, y)$ is the magnitude of the $i$ th component, $f_{i}$ is the $i$ th frequency component, and $\varphi_{i}(x, y)$ is the phase of the ith component at the position $x, y$. To obtain an "activation map" at time $t, S(x, y, t)$ was calculated from the magnitude and the phase of a frequency of the ith component. We refer to the activation map at the orientation-specific frequency as the isoorientation map (Fig. 1). The temporally encoded iso-orientation map obtained from the sinusoidal curve fitting is essentially "differential" map. Both fMRI and OIS iso-orientation maps were determined by correcting for delays related to hemodynamic responses (see Results). The correlation of the fMRI time course with a reference waveform could be calculated (Boynton et al., 1996; Engel et al., 1997) to set a statistical threshold. Correlation value normalized by a reference waveform (or statistical value) is not linearly related to the magnitude of signal changes. Because it is necessary to use absolute signal changes for determining percentage change, signal-to-noise ratio (SNR), contrast-to-noise ratio (CNR), and reproducibility, all functional images were shown without any statistical threshold.

Quantitative analysis of iso-orientation maps. Several quantitative analyses were performed within a region of interest (ROI): percentage signal change, SNR, CNR, intercolumnar distance, reproducibility, stimulus spatial frequency dependency, linear correlation, overlap, and density of singularities. To calculate SNR, magnitude at the orientation-specific frequency was divided by $\mathrm{SD}$ of magnitudes across frequencies above the orientation-specific frequency (Warnking et al., 2002). CNR is defined as the ratio of SNR within the activation area to that within the non-active area. Because the ROI of the activation area contains singularities, our $\mathrm{CNR}$ may be underestimated. Intercolumnar distance in the isoorientation maps was determined within a $5 \times 5 \mathrm{~mm}$ activation area by autocorrelation analysis (Duong et al., 2001; Fukuda et al., 2005). To compare images obtained from different modalities, global subtraction was performed on all fMRI data to remove a slow signal fluctuation, and spatial filters (Table 1) were applied to remove spatial inhomogeneity. Properties of the filter were determined by the difference of two Gaussian functions

$$
f(x)=e^{x^{2} / 2 \sigma_{1}^{2}}-e^{x^{2} / 2 \sigma_{2}^{2}},
$$

where $\sigma_{1}$ and $\sigma_{2}$ determine low-pass frequency and high-pass frequency, respectively. Singularity was visually determined in reference to magnitude and phase maps and counted within the maps of $5 \times 5 \mathrm{~mm}$.

Statistics. Data are presented as mean $\pm 1 \mathrm{SD}$, and statistical significances $(p<0.05)$ were evaluated by Student's $t$ test and multiple comparisons of Fisher's protected least significant difference after ANOVA. $p$ value of correlation coefficient was evaluated by the Student's $t$ test after Bonferroni's correction.

\section{Results}

\section{Slice selection and image coregistration based on} intracortical veins

Intracortical veins were non-invasively visualized for fMRI slice selection and for coregistration of anatomical and functional images acquired by different MRI methods. Anatomical landmarks (Fig. 2A) were determined with a $3-\mathrm{D}$ vessel-weighted imaging technique (venogram, $\sim 78 \mu \mathrm{m}$ isotropic resolution). In a coronal 2-D reconstruction of the dataset (Fig. $2 B$ ), most intracortical emerging veins appear as dark lines perpendicular to the pial surface. This pattern of intracortical vessels was used to select a tangential imaging slice along the marginal gyrus, which contains a relatively large flat section of primary visual cortex. In a tangen- 
tial 2-D reconstruction of the same dataset (Fig. 2C), intracortical veins appear as dark spots, especially within the ROI (a $5.8 \times 6.5 \mathrm{~mm}$ red box in Fig. $2 C$ ), which is the area we used for determining correspondence between fMRI and OIS maps. A single-slice 2-D anatomic image (Fig. $2 D$ ) was also acquired with the same FOV, slice thickness, and position as fMRI for coregistration between fMRI and anatomic images. A single image from the EPI dataset acquired for fMRI (Fig. 2E) again shows intracortical veins as dark spots (see ROI). Visual comparisons of vesselweighted anatomical reference images with EPI data showed that the center positions of dark spots did not shift by $>150$ $\mu \mathrm{m}$, which is equivalent to the size of one pixel before zero padding. Thus, we concluded that good coregistration was achieved within the ROI (Fig. $2 F-H$ ).

\section{Temporally encoded iso-orientation CBV-weighted fMRI maps}

To efficiently map iso-orientation columns with fMRI, we used MION as a contrast agent and adopted a temporally encoded method in which data are collected during continually cycled stimulation (Engel et al., 1997; Kalatsky and Stryker, 2003); the response to the orientationspecific stimulation cycle can be decomposed from the other frequency components by Fourier analysis. Because CBV-weighted fMRI signals were obtained during $80 \mathrm{~s}$ cyclical stimulation, the peak of the fMRI response specific to a particular orientation can therefore be observed every $80 \mathrm{~s}$ (Fig. 3A); a power spectrum of CBV-weighted fMRI signal (Fig. $3 B$ ) showed a remarkable peak at the orientation-specific stimulation frequency $[1 /(80 \mathrm{~s})=0.0125 \mathrm{~Hz}]$. In contrast, the response at the frequency corresponding to each orientation stimulation cycle $[1 /(10$ s $)=0.1 \mathrm{~Hz}$, orientationnonspecific stimulation frequency] was small because continuous stimulation saturates the orientation-nonspecific response and eventually attenuates the power at the orientation-nonspecific frequency. To calculate the percentage change of the orientation-specific frequency component for $\mathrm{CBV}$-weighted $\mathrm{fMRI}$, we divided the magnitude at the orientation-specific frequency by the sum of magnitudes at direct current and orientation-nonspecific frequencies. The average CBV-weighted orientation-specific signal change in a single $800 \mathrm{~s}$ scan was $0.62 \pm 0.23 \%$ ( $n=5$ cats $)$ in marginal gyrus, which is consistent with values from a previous report $[\sim 0.7 \%$ in block design stimulation; note that orientation-nonspecific CBVweighted $\mathrm{fMRI}$ response is significantly larger, $\sim 2 \%$ (Zhao et al., 2005)]. Peaks also appear at several other frequencies (Fig. 3B); components less than $\sim 0.01 \mathrm{~Hz}$ (e.g., arrowhead 1) result from slow baseline drift, whereas those between $\sim 0.02$ and $0.1 \mathrm{~Hz}$ (e.g., figures.
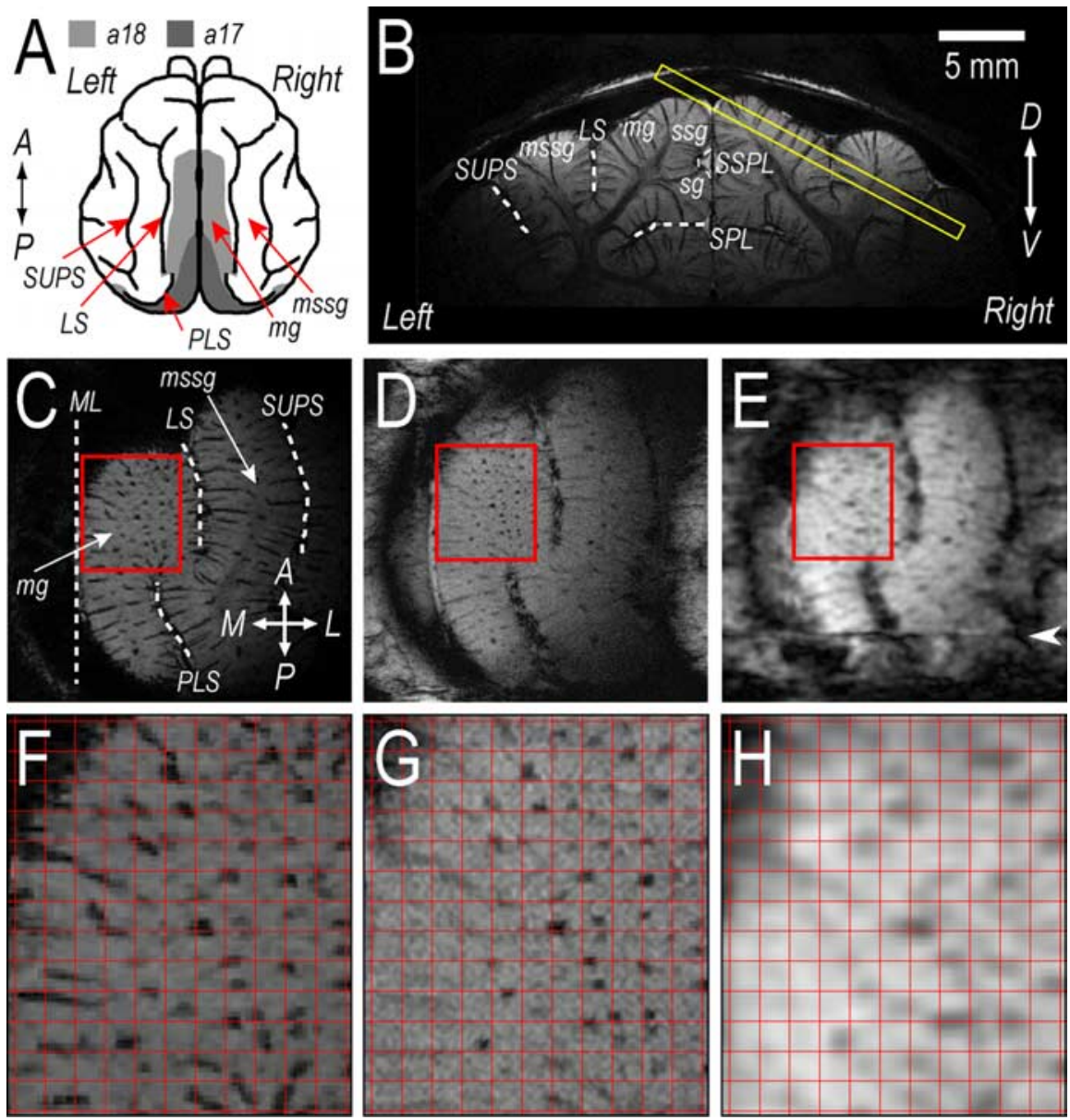

Figure 2. Selection of imaging slice for $\mathrm{fMRI}$ and comparison of venographic, anatomical reference, and functional images for distortion. $\boldsymbol{A}$, Schematic drawing of the dorsal view of cat brain. Light and dark gray areas indicate the location of areas 18 and 17 , respectively. $\boldsymbol{B}$, Coronal view (1 $\mathrm{mm}$ thickness) reconstructed from a 3-D venogram. Minimum intensity projection was performed to enhance the contrast of venous vessels, which appear as dark lines. From the 3-D venogram, the position of 1-mm-thick slice yellow rectangle in $\boldsymbol{B}$. The minimum intensity projection was reconstructed from 3-D venographic data and covers a thickness of 1 $\mathrm{mm}$, corresponding to the functional imaging slice. $\boldsymbol{D}$, Anatomical vessel-weighted reference for fMRI studies. The 1-mm-thic and anatomic images. Note that the 2-D reconstruction ( $\boldsymbol{C}$ ) and the anatomical image (D) show good agreement. $\boldsymbol{E}$, An EPI from the 作 $0.5 \times 0.5 \mathrm{~mm}$. Illustrative data in Figures $2-6$ and 8 were all obtained from the same cat. A, Anterior; $P$ , suprasplenial sulcus; SPL, splenial sulcus; SUPS, suprasylvian sulcus; mg, marginal gyrus; mssg, middle suprasylvia gyrus; ssg, suprasplenial gyrus; sg, splenial gyrus; a18, area 18; a17, area 17. The same abbreviations are used in subsequent

arrowheads 3, 4) are related to vasomotion (Mayhew et al., 1996; Obrig et al., 2000). The frequency of respiration $(\sim 0.3 \mathrm{~Hz})$ and cardiac pulsation $(\sim 3 \mathrm{~Hz})$ were out of range for this spectrum because of the $2 \mathrm{~s}$ sampling rate. However, they were aliased into the spectrum (e.g., arrowheads 6, 7). Activation maps at these frequencies were computed using Equation 1 at the $13 \mathrm{~s}$ time point in the $80 \mathrm{~s}$ stimulation cycle (see Materials and Methods). Orientation column-like patterns are seen only at the orientation-specific frequency (Fig. 3C). We will refer to activation maps at the orientation-specific frequency as iso-orientation maps.

Magnitude (Fig. 4A) and phase (Fig. 4B) maps at the orientation-specific frequency encode orientation tuning 
A

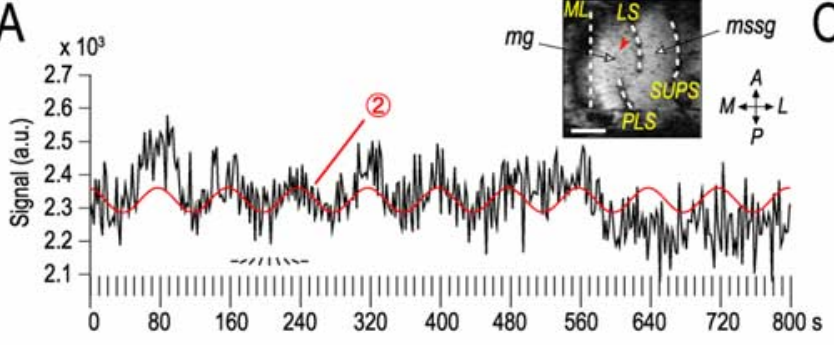

$\mathrm{B}$

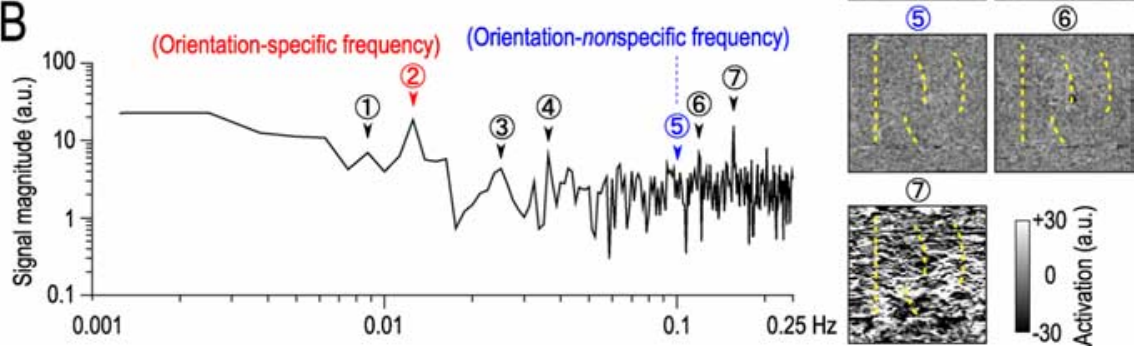

Figure 3. CBV-weighted fMRI responses to continuous orientation-specific cyclical stimulation with temporal encoding. Example was obtained from one single $800 \mathrm{~s} s c a n$. $A$, Time course of (BV-weighted fMRI signal obtained from a single pixel, which is indicated by arrowhead on the EPI (inset). Scale bar, $5 \mathrm{~mm}$. Red overlay (2) is the orientation-specific modulation $(0.0125 \mathrm{~Hz})$. Schematic representations of one cycle of stimulus orientations [from $0^{\circ}$ (horizontal) to $157.5^{\circ}$, in $22.5^{\circ}$ increments] are shown for the $160-240$ s period. $\boldsymbol{B}$, Power spectrum of the signal shown in $\boldsymbol{A}$ with the same arbitrary units (a.u.). $\boldsymbol{C}$, Activation maps for several frequencies (arrowheads $1-7$ in $\boldsymbol{B}$ ). Images in the $\boldsymbol{A}$ inset and $\boldsymbol{C}$ are shown in the same scale.
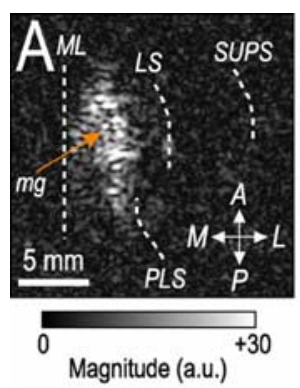

D

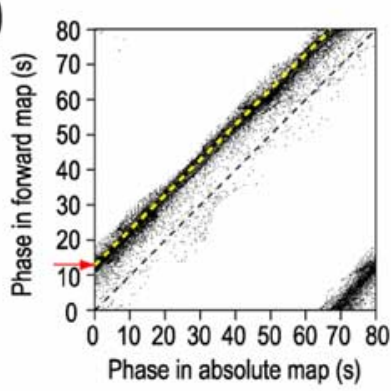

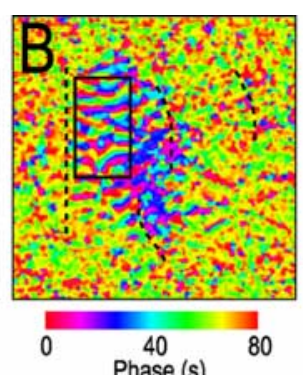
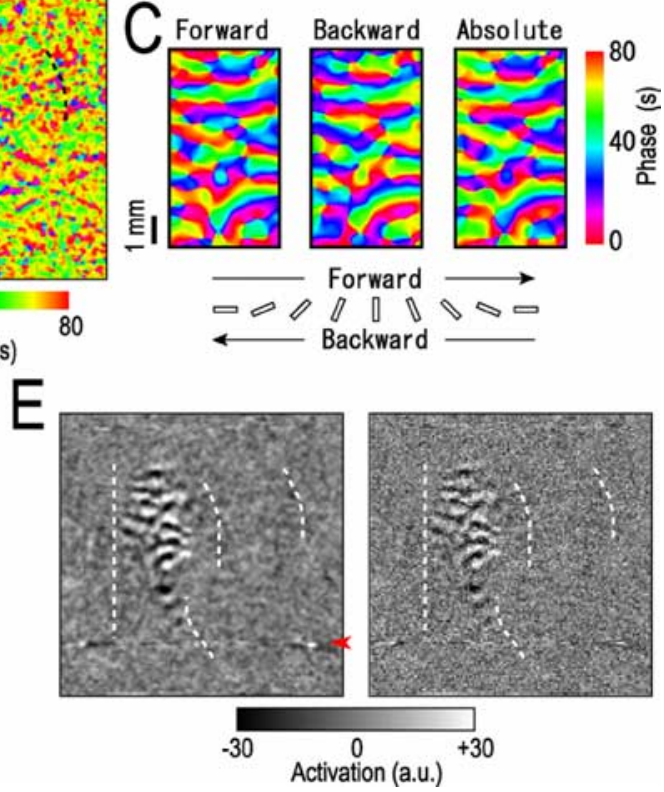

Figure 4. Assignment of stimulus orientation to temporally encoded orientation maps. Example is from CBV-weighted fMRI with two single 800 s scans: one for forward stimulation and the other for backward stimulation. $\boldsymbol{A}, \boldsymbol{B}$, Magnitude and phase maps for the orientation-specific stimulation frequency $(0.0125 \mathrm{~Hz})$, respectively. The rectangle on the phase map (forward stimulation) is enlarged in C. For convenience, phase is expressed as hemodynamic response time (seconds). C, Phase maps for forward and time-reversed backward stimulation data (left and middle, respectively) and an absolute phase map (right) calculated from the forward and backward phase maps. D, Pixelwise relationship between absolute phases and phases of forward stimulation. Average hemodynamic response time in the forward phase map is $\sim 13$ s relative to the absolute map (indicated by red arrow on vertical axis). $\boldsymbol{E}$, The iso-orientation map of $0^{\circ}$ stimulus calculated from the magnitude $(\boldsymbol{A})$ and phase $(\boldsymbol{B})$ maps after incorporation of hemodynamic response time of $13 \mathrm{~s}$; images are generated both before $(128 \times 128$; right $)$ and after $(512 \times 512$; left $)$ interpolation for comparison. The arrowhead in this map indicates a streak artifact presumably caused by a large frequency shift or by a large vessel on the lateral sulcus. Images $\boldsymbol{A}, \boldsymbol{B}$, and $\boldsymbol{E}$ are shown in the same scale. a.u., Arbitrary units.

strength and orientation preference for each pixel, respectively. Hemodynamic response is always delayed from the stimulation onset time, inducing a shift in phase values (referred to as hemodynamic response time). To accurately assign orientation prefer-

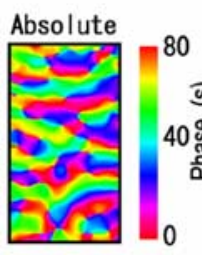

ence to phase maps, the hemodynamic response time must be determined. The hemodynamic response time can be determined using two different orientation presentation orders (Kalatsky and Stryker, 2003): one is the "forward" direction starting at $0^{\circ}$ and increasing in orientation by $22.5^{\circ}$; the other is the "backward" direction starting at $157.5^{\circ}$ and decreasing in orientation by $22.5^{\circ}$ (Fig. $4 \mathrm{C}$ schematic). The hemodynamic response time is the same in the forward and time-reversed backward stimulation data, but its direction is opposite; therefore, the effect of the hemodynamic response time can be removed from the phase maps. By averaging phase maps of these forward and backward data (Fig. 4C, left and middle, respectively), an absolute phase map (right), in which the $0^{\circ}$ stimulus presentation corresponds to a time of 0 s (i.e., no delay), can be generated. The difference between absolute phase (Fig. $4 C$, right) and phase in forward or time-reversed backward data (Fig. 4C, left or middle) indicate the hemodynamic response time. To determine the distribution of hemodynamic response times, the forward phase map was compared with the absolute map on a pixel-by-pixel basis (Fig. 4D). We found that the hemodynamic response time was typically $\sim 13 \mathrm{~s}$ in CBV-weighted fMRI with our continuous stimulation paradigm (Fig. $4 D$, indicated by a red arrow on vertical axis), which corresponds to the time-to-peak of hemodynamic response. Most pixels are distributed equally across the dotted yellow line, which has been shifted $13 \mathrm{~s}$ from the dashed diagonal, suggesting that hemodynamic response times are similar across pixels. Similar average hemodynamic response time and distribution were also observed in OIS data (data not shown). Because the hemodynamic response time is almost spatially homogeneous, the average hemodynamic response time across pixels can be used to assign stimulus orientation. This method was chosen for the current study and used to correct the hemodynamic response times for all fMRI and optical imaging signals (Table 1). In most studies, $13 \mathrm{~s}$ was used as the hemodynamic response time; the isoorientation map of $0^{\circ}$ stimulus orientation presented between 0 and $10 \mathrm{~s}$ was obtained from the $0.0125 \mathrm{~Hz}$ signals at the time of $13 \mathrm{~s}$ in the $80 \mathrm{~s}$ stimulation cycle; that of the $22.5^{\circ}$ stimulus orientation presented between 10 and $20 \mathrm{~s}$ was obtained from the $0.0125 \mathrm{~Hz}$ signals at $23 \mathrm{~s}$, etc. Note that errors in hemodynamic response time will lead to errors in orientation assignment; however, $4 \mathrm{~s}$ error (i.e., $\pm \mathrm{TR})$ will result in only $(4 \mathrm{~s} / 80 \mathrm{~s}$ 

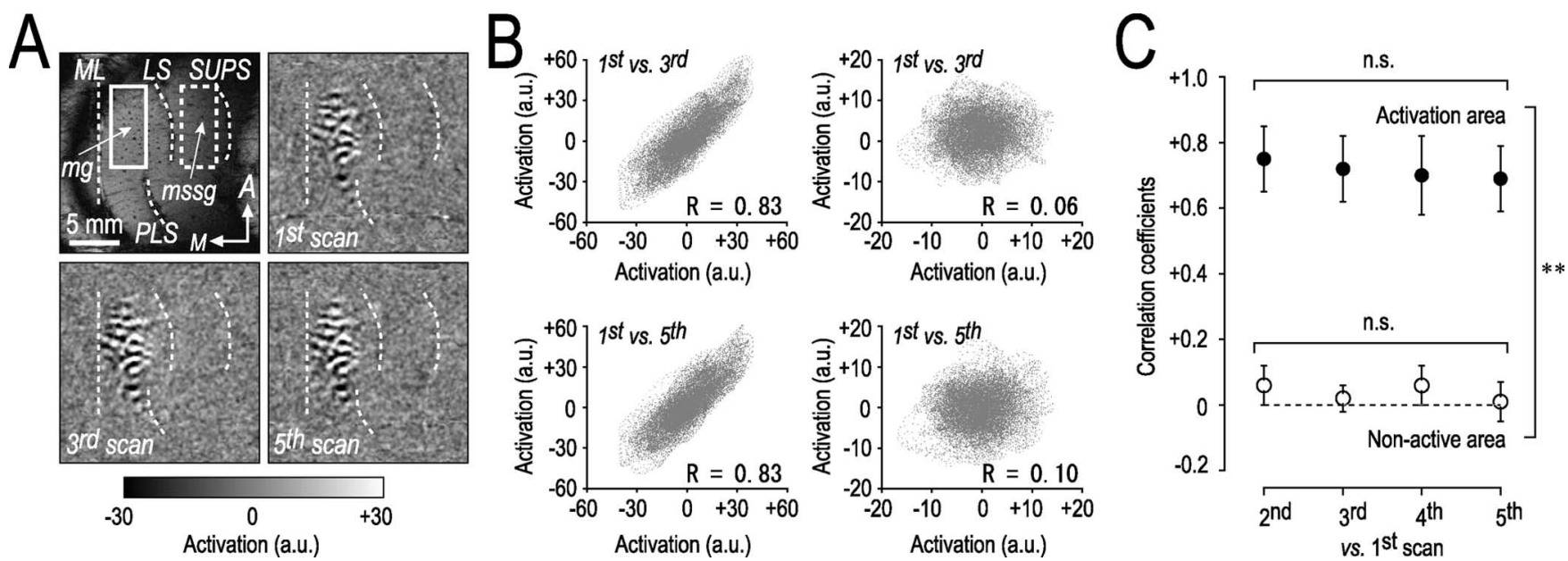

Figure 5. Reproducibility of temporally encoded CBV-weighted fMRl iso-orientation maps. $A$, Maps of $0^{\circ}$ stimulus. Each map was obtained from one single $800 \mathrm{sscan}$. Solid and dotted rectangles on the vessel-weighted anatomical reference image (top left) are ROIs selected for quantitative comparison between the activation area and the nonactive area, respectively. $\boldsymbol{B}, S$ catter plots for the $0^{\circ}$ iso-orientation map from the first scan versus those of the third and fifth scans (left, activation area; right, nonactive area). C, Correlation coefficients obtained from pixelwise map comparisons across five successive scans. Error bars are \pm 1 SD ( $n=5$ cats). ${ }^{* *} p<0.01 ; n$. s., not significant; a.u., arbitrary units.

cycle) $\times 180^{\circ}=9^{\circ}$ error, which is relatively small compared with our orientation resolution of $22.5^{\circ}$.

After correcting for the $13 \mathrm{~s}$ hemodynamic response time, we then calculated the iso-orientation map of $0^{\circ}$ stimulus presented between 0 and $10 \mathrm{~s}$ from the magnitude $(A)$ and corrected phase $(B)$ maps (Fig. $4 E$, right and left, before and after interpolation, respectively). Interpolation of raw $128 \times 128$ matrix data into $512 \times 512$ matrix did not change the appearance of functional maps. Prominent patchy and band-like activation patterns were only seen in marginal gyrus. Black patches in the $0^{\circ}$ activation maps indicate a decrease in CBV-weighted fMRI signals and thus an increase in CBV changes during $0^{\circ}$ stimulation. The average interval between the black patches or bands in the marginal gyrus was $1.2-1.6 \mathrm{~mm}(1.4 \pm 0.2 ; n=5$ cats $)$, which is similar to the distance between iso-orientation columns (1.2-1.4 $\mathrm{mm})$ reported in a 2-DG study (Lowel et al., 1987). These observations would indicate that these patches and bands likely represent isoorientation columns.

\section{Reproducibility and selectivity of CBV-weighted fMRI maps}

To determine the reproducibility of activation patterns obtained from the continuous stimulation paradigm, we compared the iso-orientation maps obtained from five different $800 \mathrm{~s}$ CBVweighted fMRI scans. CNR (see Material and Methods) of the orientation-specific CBV-weighted fMRI signal was $2.1 \pm 0.6$ ( $n=5$ cats) in a single scan, which is sufficiently high to perform scan-by-scan comparisons. Activation patterns corresponding to $0^{\circ}$ stimulus orientation were consistent across repeated scans (Fig. 5A). Pixelwise correlation analysis (Fig. 5B) showed that signal changes within the primary visual cortex (solid white "activation area" rectangle in Fig. 5A) are highly reproducible $(r=$ 0.83 for first vs third scan and 0.83 for first vs fifth scan; $p<$ 0.001). In comparison, the correlation coefficient within a brain region outside of the primary visual cortex (dotted white "nonactive area" rectangle in Fig. $5 A)$ is close to $0(r=0.06$ for first vs third scan and 0.10 for first vs fifth scan). These observations were consistent for all eight orientations. The average pixelwise correlation coefficient for all eight orientations within ROI in the activation area (e.g., $r=0.75 \pm 0.10$ at first vs second scans; $n=5$ cats) was significantly higher (ANOVA, $F_{(1,32)}=641.430 ; p=$ $9.67 \times 10^{-23}$ ) than those in the non-activation area (e.g., $r=$
$0.06 \pm 0.06$ at first vs second scans; $n=5$ cats) across five scans (Fig. 5C). The correlation coefficients were not significantly different across five scans (ANOVA, $F_{(3,12)}=2.207, p=0.140$ for activation area; $F_{(3,12)}=2.093, p=0.155$ for non-active area), suggesting that a similar activation pattern is observed for $\sim 67$ min (i.e., $800 \mathrm{~s} / \mathrm{scan} \times 5$ scans).

To confirm that the observed activation patterns are in fact within area 18, we obtained CBV-weighted fMRI responses to both low $\left(0.1 \mathrm{cycle}^{\circ}\right)$ and high $\left(0.3 \mathrm{cycle}^{\circ}\right)$ spatial frequency stimulation (Movshon et al., 1978) (Fig. 6A). Iso-orientation maps obtained from one $800 \mathrm{~s}$ scan for all eight stimulation orientations show a smooth change in activation pattern with a change in orientation (top row), and the patterns are complementary at half the orientation-specific stimulation cycle [e.g., compare $0^{\circ}(13 \mathrm{~s})$ and $90^{\circ}(53 \mathrm{~s})$ maps]. Most orientation-specific signals in marginal gyrus with low-spatial-frequency stimulation (top row) were not present with high-spatial-frequency stimulation (bottom row), which verifies that these signals originate from area 18. In contrast, activation in the posteromedial part of the marginal gyrus was stronger during high-spatial-frequency stimulation, suggesting an association with area 17 . It should be noted that the area 17 in this slice is perpendicular to the cortical surface, so the assignment of stimulus orientation is distorted because of contributions of neighboring columns in the $1 \mathrm{~mm}$ slice thickness; thus, typical black and white patterns are not represented.

The distinct response to low and high-spatial-frequency stimulation was much clearer in the magnitude maps (Fig. 6B). To quantitatively determine how spatial frequency stimulation affected the magnitude of signal change, we compared histograms of magnitude within the $2.1 \times 2.1 \mathrm{~mm}$ ROI shown in Figure $6 B$ (red and green boxes). As shown in Figure 6C, in area 18, lowspatial-frequency stimulation induced higher signal changes than high-frequency stimulation (compare red solid line vs red dotted line), whereas in area 17, high-frequency stimulation induced higher signal changes than low-frequency stimulation (compare green dotted line vs green solid line). This was consistently observed in all five cats tested. In area 18, the average cumulative curve obtained with low-spatial-frequency stimulation was significantly shifted to the right (ANOVA, $F_{(1,98)}=11.849 ; p=$ 0.001 ) compared with the curve from high-frequency stimula- 

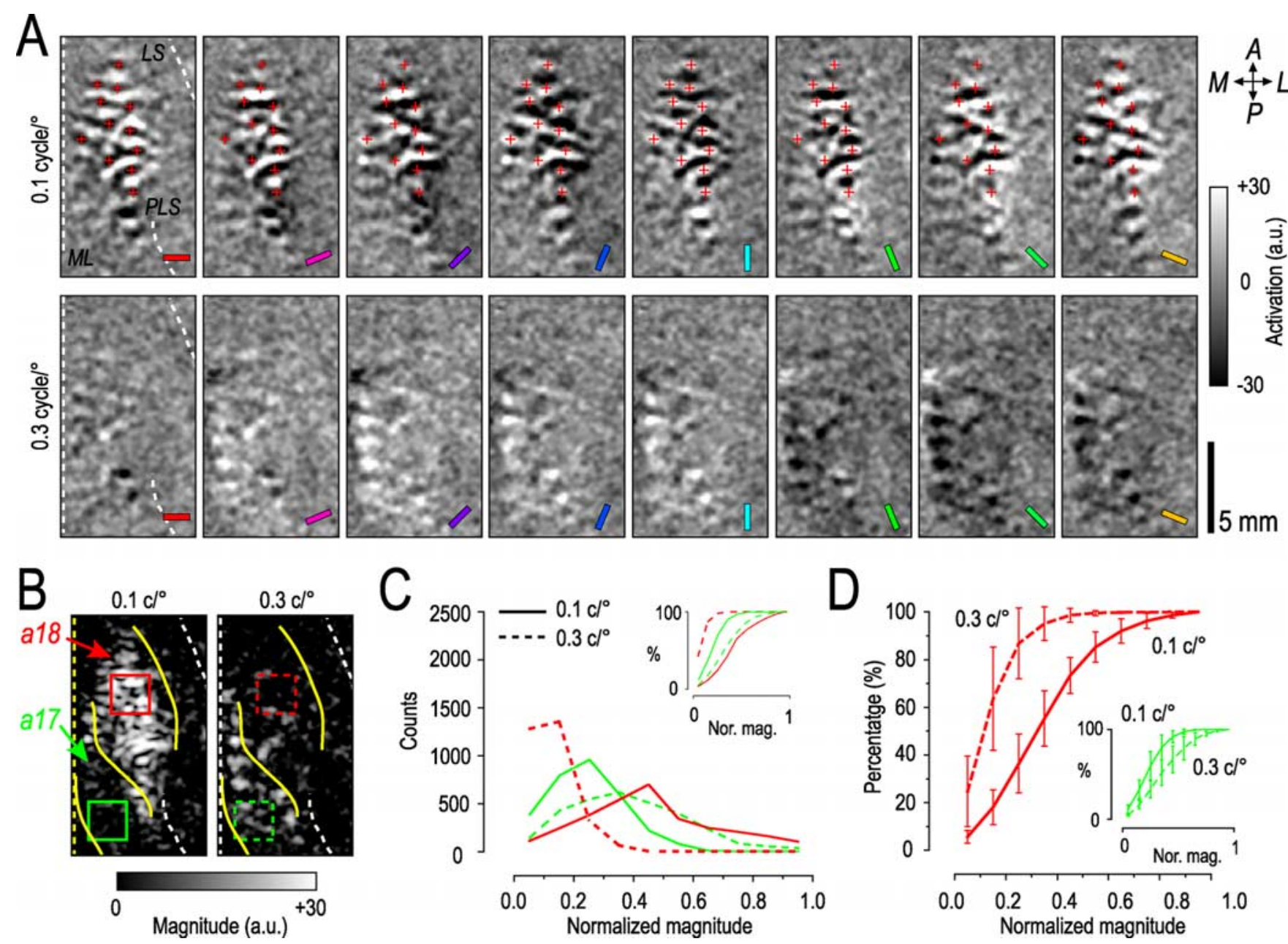

Figure 6. Orientation and spatial frequency-specific responses in primary visual cortex of CBV-weighted fMRI. Iso-orientation maps were obtained from one single $800 \mathrm{~s} s c a n$ for each spatial frequency. $A$, Iso-orientation maps for the eight stimulation orientations. Responses to low $\left(0.1\right.$ cycle $\left./{ }^{\circ}\right)$ and high $\left(0.3 \mathrm{cycle} /{ }^{\circ}\right)$ spatial frequency stimulation are shown in the top and bottom rows, respectively. For easier comparison of the orientation specificity, plus signs indicating increases of CBV in the $0^{\circ}$ iso-orientation map are overlaid on other panels. $\boldsymbol{B}$, Magnitude maps of the orientation-specific response for low- and high-spatial-frequency stimulation. Yellow solid lines trace approximate functional borders of areas 17 and 18 . Red and green boxes indicate ROIs for histogram analysis in areas 18 and 17, respectively. Images in $\boldsymbol{A}$ and $\boldsymbol{B}$ are shown in the same scale. $\boldsymbol{C}$, Histograms of response magnitude to low- and high-spatial-frequency stimulation shown in B. Magnitudes are normalized to the maximum responses to low-spatial-frequency stimulation in area 18 and to high-spatial-frequency stimulation in area 17 (red solid line for responses to 0.1 cycle $/{ }^{\circ}$ in area 18 ; red dotted line for responses to 0.3 cycle $/{ }^{\circ}$ in area 18 ; green solid line for responses to 0.1 cycle $/{ }^{\circ}$ in area 17 ; green dotted line for responses to 0.3 cycle $/{ }^{\circ}$ in area 17$)$. Bin width is 0.1. Inset, The same data are plotted as cumulative percentage histograms. D, Average cumulative percentage histograms for five cats within area 18 and (inset) for three cats within area 17 (in 2 cats, area 17 was not within the field of view). Error bars are \pm 1 SD. a.u., Arbitrary units.

tion in the same area (Fig. $6 C$, inset, $D$ ); this demonstrates that the low-spatial-frequency stimulation induced higher magnitude changes. In area 17, the average cumulative curve obtained with high-spatial-frequency stimulation was not statistically different (ANOVA, $\left.F_{(1,58)}=2.566 ; p=1.115\right)$ from the curve with lowfrequency stimulation (Fig. $6 D$, inset). These results indicate that the activation patterns observed in the low-spatial-frequency study are primarily attributed to area 18 of visual cortex (Movshon et al., 1978).

\section{$\mathrm{CBV}$-weighted versus $\mathrm{CMRO}_{2}$-based fMRI iso-orientation maps}

Our CBV-weighted iso-orientation fMRI maps clearly show reproducible and selective activation patterns. To examine the PSF of hemodynamic response, we compared CBV-weighted isoorientation $\mathrm{fMRI}$ maps with $\mathrm{CMRO}_{2}$-based $\mathrm{fMRI}$ iso-orientation maps. Metabolism-based fMRI signals (Kim et al., 2000) can be extracted from blood oxygenation level-dependent (BOLD) re- sponse by the intravenous infusion of sNP (Nagaoka et al., 2006), which suppresses evoked hemodynamic (CBF and CBV) responses without changing evoked spiking activity (Fukuda et al., 2006). As seen from the images (Fig. 7A) (for another example, see supplemental Fig. 1, available at www.jneurosci.org as supplemental material), most patches with higher $\mathrm{CMRO}_{2}$ changes have higher CBV responses; iso-orientation maps of the $\mathrm{CMRO}_{2}$ based fMRI signal were significantly correlated to those of CBVweighted fMRI $(r=0.71, p<0.001$ in Fig. $7 B ; r=0.66 \pm 0.10$, $n=4$ cats in Fig. $7 C$ ). Some mismatch is likely attributable to poor CNR of the single-scan $\mathrm{CMRO}_{2}$-based fMRI signal (1.4 \pm 0.3 ), which is significantly smaller $(p=0.0097$, two-tail paired $t$ test; $n=4$ cats) than CNR of the single-scan CBV-weighted fMRI $(1.9 \pm 0.3)$. This is also supported by average correlation coefficient between first versus second scans, which yields $0.32 \pm 0.26$ $(n=4)$ for $\mathrm{CMRO}_{2}$-based fMRI and $0.68 \pm 0.13$ for CBVweighted fMRI. To increase the CNR of $\mathrm{fMRI}$ signals, all repeated scans were averaged (Fig. 7C). The significant positive correlation 
between $\mathrm{CBV}$-weighted and $\mathrm{CMRO}_{2}$ based fMRI maps suggests that (1) the PSF of $\mathrm{CBV}$ response is narrower than the intercolumn distance as discussed in Figure $1 B$, and (2) active columns in CBVweighted fMRI maps likely indicate sites of increased neuronal activity because oxidative metabolism is known to be highly correlated with neuronal activity (Thompson et al., 2003).

\section{CBV-weighted fMRI versus optical imaging iso-orientation maps}

To more convincingly determine whether the observed bands and patches in CBVweighted fMRI maps mark the actual sites of increased neuronal activity, we recorded OIS data after we collected CBVweighted fMRI data ( $n=6$ cats). We compared the plasma-based CBV-weighted fMRI signal with CBV-weighted $570 \mathrm{~nm}$ OIS, which indicates a change in total hemoglobin, or red blood cell (RBC) counts, thus addressing a change in total blood volume at the resolution of functional columns. Previously, neural correlates to OIS in visual cortex have only been demonstrated with $\mathrm{dHb}$-weighted wavelengths (Grinvald et al., 1986; Shmuel and Grinvald, 1996; Maldonado et al., 1997; Bosking et al., 2002). However, good agreement between iso-orientation maps of 570 nm OIS and dHb-weighted OIS (Fukuda et al., 2005) (supplemental Fig. 2, available at www.jneurosci.org as supplemental material) suggests that $570 \mathrm{~nm}$ OIS is also a good neural correlate.

Functional MRI and optical images were coregistered using pial vessel patterns; because the slice chosen for fMRI studies was below the cortical surface (Fig. $8 A$, red rectangle), the pial vessel pattern above the fMRI slice (Fig. $8 \mathrm{~A}$, green rectangle) was visualized using a $2-\mathrm{D}$ MR image reconstructed from the $3-\mathrm{D}$ venogram (Fig. $8 B$ ). Then, the optical image (Fig. $8 C$ ) was linearly transformed manually so that pial vessel patterns matched the MRI patterns within the ROI (Fig. $8 B, C$, green boxes). Based on visual inspection after coregistration, we estimate that any remaining mismatch between pial vessel locations is $\leq 200 \mu \mathrm{m}$ within the $5.8 \times 6.5$ mm ROI (Fig. 8, compare $D, E$ ); this mismatch may be attributable to a slight difference in imaging plane orientations between MRI and optical imaging. Taking into account the possible coregistration error between MR anatomical images and fMRI data, the overall coregistration error between fMRI maps and OIS maps is $\leq 350 \mu \mathrm{m}$. Because the width of isoorientation columns (i.e., half of the inter-
A
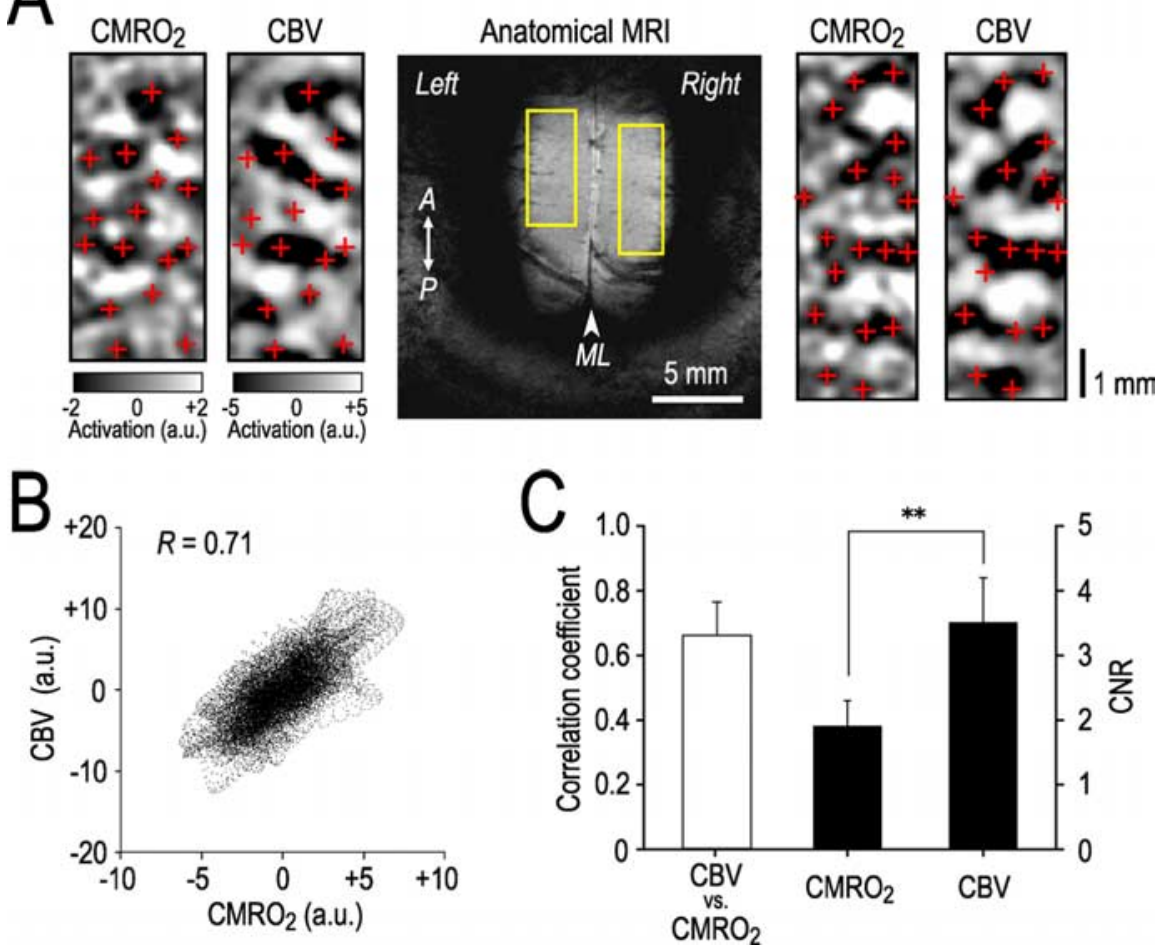

Figure 7. Iso-orientation maps obtained from $\mathrm{CMRO}_{2}$-based fMRI versus CBV-weighted fMRI. All three to five repeated $800 \mathrm{~s}$ scans were averaged for sensitivity improvement. $\boldsymbol{A}$, Maps of $0^{\circ}$ stimulus orientation for $\mathrm{CMRO}_{2}$-based fMRI and $\mathrm{CBV}_{\text {-weighted }}$ fMRI obtained from ROIs (yellow rectangles) on left and right hemispheres shown in anatomical MR image. For easier comparison, plus signs indicating increase in deoxygenation for $0^{\circ}$ stimulation in $\mathrm{CMRO}_{2}$-based fMRI maps are overlaid on CBV-weighted fMRI maps. $\boldsymbol{B}$, Scatter plots for the maps shown in $\boldsymbol{A}$ (plots are obtained from both hemispheres). A decrease in CBV-weighted fMRI signal indicates an increase in $\mathrm{CBV}$, and a decrease in $\mathrm{CMRO}_{2}$-based $\mathrm{fMRl}$ signal indicates an increase in deoxygenation. $\boldsymbol{C}$, Left white bar, Average correlation coefficients in comparisons of $\mathrm{CMRO}_{2}$-based $\mathrm{fMRI}$ versus $\mathrm{CBV}$-weighted fMRI maps. Middle and right black bars, CNR differences between the two fMRI techniques. Error bars are \pm 1 SD ( $n=4$ cats). ${ }^{* *} p<0.01$. CNRs of $\mathrm{CMRO}_{2}$-based and CBV-weighted fMRI are after average for three to five and five scans, respectively. a.u., Arbitrary units.
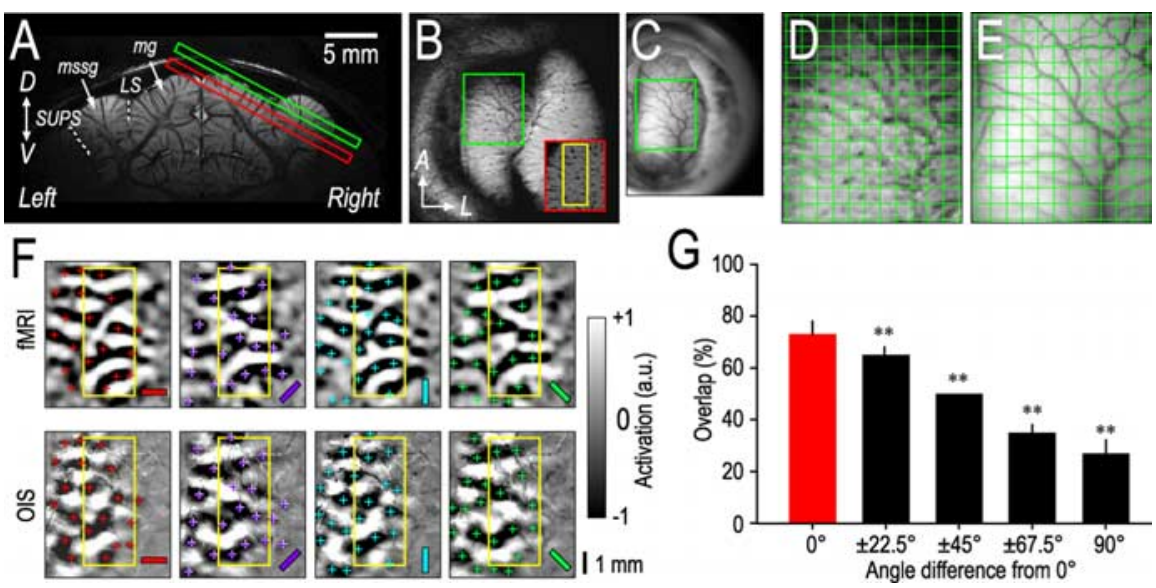

Figure 8. Comparison between iso-orientation maps obtained with CBV-weighted fMRI and $570 \mathrm{~nm}$ 0IS. All repeated $800 \mathrm{~s}$ scans were averaged for sensitivity improvement. $\boldsymbol{A}$, Slice positions for $\mathrm{FRI}$ (red rectangle) and for MR venographic image of pial vessel patterns (green rectangle) on coronal view (1 $\mathrm{mm}$ thickness) reconstructed from a 3-D venogram. $\boldsymbol{B}$, Dorsal view of the MR venographic image at the location of green rectangle in $\boldsymbol{A}$. Inset, MR venographic image from a location matched to fMRI maps (red rectangle in $\boldsymbol{A}$ ); the yellow rectangle (at the same location as the yellow rectangles in $\boldsymbol{F}$ ) indicates the region perpendicular to emerging veins (dark spots) and is chosen for quantitative comparisons (inset image is the same as Fig. 2 F). C, Pial vessel patterns obtained by optical imaging at a $570 \mathrm{~nm}$ wavelength. $\boldsymbol{A}$-C are shown in the same scale. $\boldsymbol{D}, \boldsymbol{E}$, Enlarged coregistered regions (5.8 $\times$ $6.5 \mathrm{~mm}$ boxes on $\boldsymbol{B}$ and $\boldsymbol{C}$ ) for detailed comparison. Grids are $0.5 \times 0.5 \mathrm{~mm}$. $\boldsymbol{F}$, Iso-orientation maps of (BV-weighted fMRI (top row) and OIS (bottom row) from one cat after pial vessel coregistration showing $0,45,90$, and $135^{\circ}$ results only. For easier comparison, plus signs indicating the increases of CBV in individual $570 \mathrm{~nm}$ 0IS maps are overlaid on fMRI maps. G, Overlap of columns in fMRI and OIS binary maps (percentage by number of pixels). Error bars are $\pm 1 S D(n=6) .{ }^{* *} p<0.01$ (compared with $0^{\circ}$ difference). a.u., Arbitrary units. 

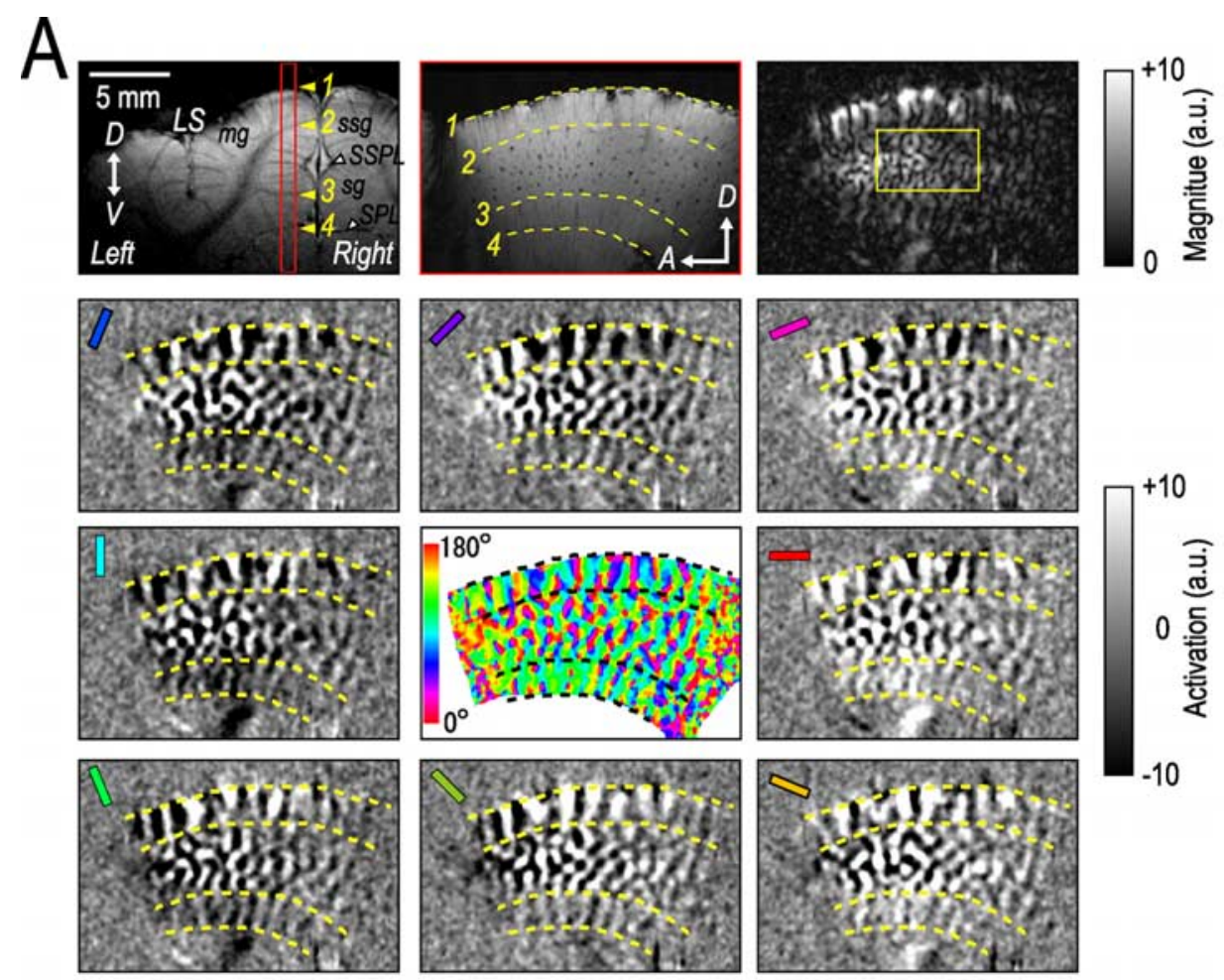

$B$
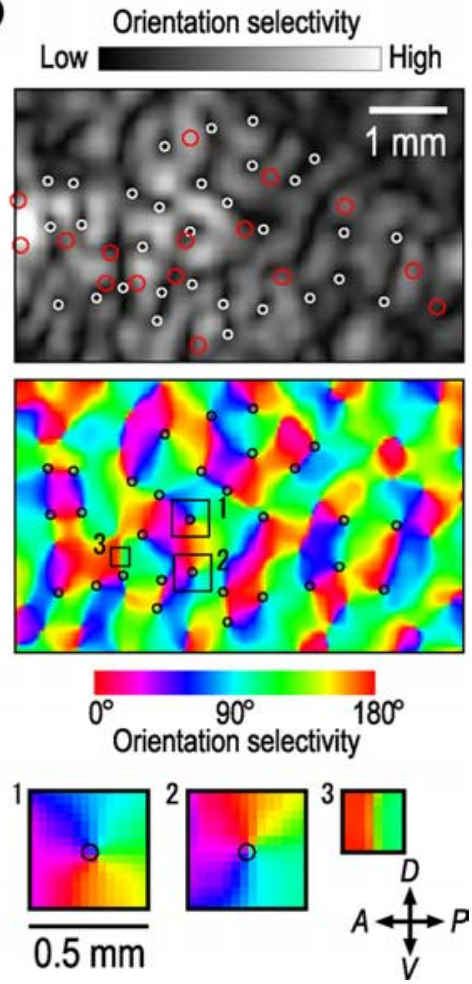

Figure 9. Columnar mapping inside cortical sulcus with (BV-weighted fMRI. Two repeated $800 \mathrm{~s}$ scans were averaged for sensitivity improvement. $\boldsymbol{A}$, Top row, Coronal view of vessel-weighted anatomical reference image shows the position of $\mathrm{fMRI}$ slice as red rectangle (left). Vessel patterns of the sagittal imaging slice are shown (middle). Magnitude map of orientation-specific responses are shown (right). Remaining panels, Eight iso-orientation maps are arranged around the center color-coded orientation preference map. $\boldsymbol{B}$, Grayscale magnitude map (top) and colored orientation preference map (middle) enlarged from the yellow rectangle on original map. White and black circles in these maps indicate orientation column convergences (i.e., singularities). Two pinwheel structures $(1,2)$ and a fracture-like structure (3) are enlarged further (bottom). Red circles overlaid on the magnitude map mark positions of intracortical veins. Note that dark spots and lines in the magnitude map generally do not correspond to blood vessels (red circles). The fractures are obscured in the phase map because some of the colors span a large range of orientation assignments (e.g., green represents $\sim 100-135^{\circ}$ ). a.u., Arbitrary units.

column distance) is $700 \mu \mathrm{m}$, criterion for a good congruence between $\mathrm{AMRI}$ and OIS columns will therefore appear as an overlap of $>350 \mu \mathrm{m}(>50 \%)$.

Iso-orientation maps of CBV-weighted fMRI were similar to those of $570 \mathrm{~nm}$ OIS in all six cats tested (Fig. $8 \mathrm{~F}$ ) (for another example, see supplemental Fig. 3, available at www.jneurosci.org as supplemental material); activation borders, columnar patterns (black and white patches and bands), and most loci show a good match between the two modalities. To quantify the results, we converted the iso-orientation maps of CBV-weighted $\mathrm{AMRI}$ and $570 \mathrm{~nm}$ OIS into binary images. Assuming minimal distortion of columnar shape in regions in which the image is perpendicular to intracortical emerging vessels, an ROI was appropriately chosen from the vessel-weighted MR anatomical image (Fig. $8 \mathrm{~B}$, inset, yellow rectangle) (for sizes of ROIs, see Table 1). Because of the differences in pixel resolution and signal magnitude between fMRI and OIS maps, pixelwise correlation analysis was not performed. Instead, the percentage overlap was calculated. Pixels with a value smaller than the mean value within this ROI (Fig. $8 \mathrm{~F}$, yellow rectangle) were assigned as active for a given orientation, whereas others were assigned as inactive for the same orientation. Then, we calculated the percentage of overlap for both active and inactive pixels between the OIS and fMRI binary maps from all eight orientations. There were significant differences for the percentage of overlap (ANOVA, $F_{(4,20)}=147.32 ; p=1.55 \times 10^{-14}$ ) across the stimulation angle differences from $0^{\circ}$ orientation. The highest percentage of overlap was found when the stimulus orientation was the same between OIS and fMRI maps (Fig. 8G) $\left(72.5 \pm 4.7 \% ; n=6\right.$ cats at $0^{\circ}$ angle difference; $p=2.6 \times 10^{-4}$ to $8.00 \times 10^{-5}$ compared with other angle differences). Because this value meets our criterion for good congruence $(>50 \%)$, we conclude that $\mathrm{CBV}$-weighted $\mathrm{AMRI}$ iso-orientation maps agree with OIS maps. Thus, the highest CBV-weighted fMRI signal change is expected at the site of increased neuronal activity.

\section{Mapping orientation columns inside cortical sulcus}

We demonstrated that MION-based CBV-weighted fMRI techniques can reliably map iso-orientation columns on dorsal cortical surface regions. Thus, with this highly sensitive MION-based CBV-weighted fMRI, functional columns can be mapped in cortical regions deeply embedded in sulci to which OIS imaging cannot be applied. We attempted to record orientation-specific responses on the medial bank of cortical area 17 (suprasplenial and splenial gyri) in two cats, an area that is inaccessible with OIS imaging. We successfully mapped iso-orientation columns in this area (Fig. 9A). The activation pattern was highly reproducible; the pixelwise correlation coefficient between two successive scans within a region of activity was 0.89 and $0.66(p<0.001)$ at $0^{\circ}$ iso-orientation maps for two cats. Spatially distinct activation patterns on a sagittal imaging slice appear as irregular patches and bands (Fig. 9A). Patchy patterns appear in the region between dashed lines 2 and 3, in which the imaging plane is parallel to the cortical surface and in which the vessel-weighted anatomical image shows a high density of tangential intracortical veins. Bandshaped patterns appear in regions in which the imaging plane is perpendicular to the cortical surface and in which intracortical 
veins run parallel to the slice orientation (between dashed lines 1 and 2 and between lines 3 and 4). The arrangement of isoorientation columns for all orientations $\left(0-180^{\circ}\right)$ was represented in the orientation preference map by color coding. Assignment of stimulus orientation in the regions perpendicular to the cortical surface (between dashed lines 1 and 2 and between 3 and 4) was distorted because of contributions of neighboring columns in the $1 \mathrm{~mm}$ slice thickness. Similarly, the assignment of stimulus orientation was inaccurate in the most anterior and posterior regions because of low signal sensitivity. Detail of the orientation column structure is seen in a region selected between dashed lines 2 and 3 (rectangle in the magnitude map), an area in which signal sensitivity is high and cortical vessels run perpendicular to the slice. Consistent with findings by OIS imaging from the exposed cortical surface (marginal gyrus), the orientation preferences change abruptly at singularities (Bonhoeffer and Grinvald, 1993) and at fracture-like structures (Blasdel, 1992; Rao et al., 1997) (Fig. 9B), which appear as regions of low signal intensity in the magnitude map (dark spots and lines). There were $2.0-2.5$ singularities $/ \mathrm{mm}^{2}$ in the medial bank of area 17 $(n=2)$, which is higher than the density of singularities (1.3-1.8/ $\mathrm{mm}^{2} ; n=5$ cats) in the dorsal part of area 18 . These values are similar to results obtained from OIS studies in the dorsal region of area $17\left(2.1-2.4 / \mathrm{mm}^{2}\right)$ (Bonhoeffer et al., 1995; Rao et al., 1997 ) and area $18\left(1.2-2.1 / \mathrm{mm}^{2}\right)$ (Bonhoeffer and Grinvald, 1993; Womelsdorf et al., 2001). Our results further suggest that fMRI can be used to map submillimeter-scale functional structures without depth limitation.

\section{Discussion}

Our major finding is that CBV-based fMRI iso-orientation maps are similar to $\mathrm{CMRO}_{2}$-based fMRI and OIS maps on a columnar level; even when multiple columns are simultaneously activated, the highest signal change in CBV-weighted fMRI maps generally corresponds to the highest signal change in $\mathrm{CMRO}_{2}$-based fMRI and OIS maps. This leads us to infer that most sites of the highest orientation-selective CBV signals correspond to the sites of increased neuronal activity. Thus, the PSF of CBV response is sufficiently narrow to resolve iso-orientation columns (Fig. $1 B$, schematic profile of 1 ); the highest $\mathrm{CBV}$ changes induced by bars of $0^{\circ}\left(90^{\circ}\right)$ orientation are likely to indicate $0^{\circ}\left(90^{\circ}\right)$ orientation columns. However, this finding cannot simply be applied to neural interpretation of BOLD columnar maps because the BOLD signal is directly related to a mismatch between $\mathrm{CBF}$ and $\mathrm{CMRO}_{2}$ responses. Even if $\mathrm{CBV}$ (or $\mathrm{CBF)}$ ) and $\mathrm{CMRO}_{2}$ responses are localized to active columns (Fig. 7), the highest changes in BOLD fMRI signals may not indicate active columns (Duong et al., 2000; Cheng et al., 2001); thus, additional studies are needed.

\section{Improvement of high-resolution fMRI specificity and sensitivity}

Imaging techniques must have high specificity and sensitivity to be useful for neuroscience research. Because oxygen metabolism occurs at the site of increased neuronal activity (Thompson et al., 2003), imaging the metabolic change should yield high spatial specificity (Malonek and Grinvald, 1996). Thus, after the presentation of a stimulus, the early negative BOLD signal (referred to as an "early dip") (Kim et al., 2000), which likely indicates an early oxygen consumption change (Nagaoka et al., 2006), should provide high specificity to active cortical columns. However, to detect this early response and use the high spatial specificity of the early dip signal, images must be acquired with high spatial and temporal resolution, which results in a significant reduction in sensitivity. Although this can be overcome using a vasodilator, which induces a $\mathrm{CMRO}_{2}$-based fMRI signal (Nagaoka et al., 2006), the vasodilator cannot be easily used for human studies. Therefore, it is currently difficult to achieve sufficient sensitivity for metabolic mapping of multiple orientation columns within a reasonable time frame without the assistance of vasodilator.

To improve the specificity and sensitivity of functional signals, we injected MION, a blood plasma contrast agent. Compared with the conventional BOLD fMRI technique, MION-based CBV fMRI has high sensitivity of functional signals (Kennan et al., 1998; Mandeville et al., 1998; van Bruggen et al., 1998) and high specificity to microvessels (Mandeville and Marota, 1999; Zhao et al., 2006). Sensitivity enhancement is dependent on many parameters, including magnetic field strength and dose of contrast agent (Kim and Ugurbil, 2003). Because an increased BOLD signal at higher magnetic fields counteracts the effect of MION, $\mathrm{CBV}$-weighted $\mathrm{fMRI}$ at lower magnetic fields results in a greater improvement in sensitivity. However, even at 9.4 T, we found that functional sensitivity with $10 \mathrm{mg} \mathrm{Fe} / \mathrm{kg}$ MION was improved by $>1.6$ times over the conventional gradient-echo BOLD fMRI in the cat visual cortex (Zhao et al., 2006). The high sensitivity of MION-based CBV fMRI leads to excellent reproducibility for small functional signals (Fig. 5). Thus, CBV-weighted fMRI is a superior alternative to conventional BOLD fMRI in brain research with animal models (Dubowitz et al., 2001; Vanduffel et al., 2001; Leite et al., 2002; Fize et al., 2003; Tsao et al., 2006); this technique, however, cannot be easily used in human studies because a large amount of exogenous contrast agent is required. Alternatively, non-invasive CBV-based fMRI, such as the vascular-space-occupancy technique (Lu et al., 2003, 2004), can be used if it has sufficient sensitivity.

To further improve the specificity and sensitivity of functional signals, we used the temporally encoded method with a gradual shift in orientations during continuous stimulation. This approach has been routinely used for fMRI retinotopic mapping (Engel et al., 1994; Sereno et al., 1995; Engel et al., 1997; Tootell et al., 1998), and it was recently applied to OIS imaging to generate orientation-preference maps (Kalatsky and Stryker, 2003). There are several advantages to continuous stimulation with temporal encoding. The fMRI response after the orientation-specific stimulation cycle can be easily separated from other frequency components induced by vasomotion, respiration, heart beat, etc., by means of frequency analysis. Also, the orientation nonspecific signal induced by each stimulus presentation is almost saturated by continuous stimulation (Fig. $3 B, C$, component 5 ), essentially similar to the removal of orientation nonspecific signal, which occurs with cocktail blank subtraction (Bonhoeffer and Grinvald, 1993). Finally, the signal responds to the orientation changes in a gradual manner, which is well fitted by our cosine function model. Thus, the Fourier analysis method with continuous stimulation (Kalatsky and Stryker, 2003) is more efficient to detect functional response compared with a conventional block-design paradigm (Kim et al., 2000; Duong et al., 2001; Zhao et al., 2005). However, determining hemodynamic response time is not so straightforward for data obtained from continuous stimulation. If a pixel is activated from multiple stimulations (e.g., 0 and $90^{\circ}$ ), it will be difficult to assign orientation preference (Zepeda et al., 2004). Furthermore, if the hemodynamic response time is not spatially homogeneous, determining pixelwise hemodynamic response time to assign orientation preference would require forward and backward stimulation (Fig. 4C) (Sereno et al., 1995; Warnking et al., 2002; Kalatsky and Stryker, 2003). In our studies, however, the hemodynamic response time is similar across pixels 
in area 18 (Fig. 4D), thus eliminating the need to perform the backward stimulation.

\section{Neurovascular control at a columnar level}

The congruence of CBV-weighted fMRI maps and $570 \mathrm{~nm}$ OIS maps suggests an increase in total blood volume at a columnar level. Because CBV-weighted $\mathrm{CMRI}$ with a contrast agent and 570 nm OIS techniques are sensitive to plasma volume (Kennan et al., 1998; Mandeville et al., 1998; van Bruggen et al., 1998) and RBC count (Frostig et al., 1990; Malonek et al., 1997; Sheth et al., 2004; Vanzetta et al., 2004; Suh et al., 2005; Fukuda et al., 2006), respectively, our data can be used to obtain an insight into neurovascular control on a columnar level. During orientation-selective stimulation, RBCs might redistribute across cortical columns. According to in vivo microscopic studies (Villringer et al., 1994; Hudetz, 1997), 10-20\% of capillaries (i.e., microvessels of $\sim 5$ $\mu \mathrm{m}$ diameter) carry only plasma during resting conditions, although all of these capillaries carry a mixture of RBCs and plasma during stimulation conditions. If total microvascular blood volume remains the same over the cortex (Wei et al., 1993), it would suggest a reduction of plasma volume in active columns and an increase of plasma volume in inactive columns. However, our CBV-weighted fMRI and OIS data show that both plasma volume and RBC count increase within the same iso-orientation columns. Similar colocalization of activity-dependent changes in plasma volume and RBC count is also reported in studies comparing OIS imaging at the wavelength of the hemoglobin isosbestic points and optical imaging of intravascular plasma tracers in monkey ocular dominance column (Frostig et al., 1990), rodent barrel field (Narayan et al., 1995), and cat orientation column (Fukuda et al., 2005; Vanzetta et al., 2005). Combining our data with those of others, it can be concluded that the major mechanism of columnar-specific CBV response is not a redistribution of "RBC to plasma ratio" across cortical columns but rather dilation of microvessels within active columns. This could be explained by recently demonstrated hemodynamic regulation mechanisms via the activity of astrocytes (Zonta et al., 2003; Takano et al., 2006) and pericytes (Howarth et al., 2005).

\section{References}

Blasdel GG (1992) Differential imaging of ocular dominance and orientation selectivity in monkey striate cortex. J Neurosci 12:3115-3138.

Blasdel GG, Salama G (1986) Voltage-sensitive dyes reveal a modular organization in monkey striate cortex. Nature 321:579-585.

Bonhoeffer T, Grinvald A (1993) The layout of iso-orientation domains in area 18 of cat visual cortex: optical imaging reveals a pinwheel-like organization. J Neurosci 13:4157-4180.

Bonhoeffer T, Kim DS, Malonek D, Shoham D, Grinvald A (1995) Optical imaging of the layout of functional domains in area 17 and across the area 17/18 border in cat visual cortex. Eur J Neurosci 7:1973-1988.

Bosking WH, Crowley JC, Fitzpatrick D (2002) Spatial coding of position and orientation in primary visual cortex. Nat Neurosci 5:874-882.

Boynton GM, Engel SA, Glover GH, Heeger DJ (1996) Linear systems analysis of functional magnetic resonance imaging in human V1. J Neurosci 16:4207-4221.

Cheng K, Waggoner RA, Tanaka K (2001) Human ocular dominance columns as revealed by high-field functional magnetic resonance imaging. Neuron 32:359-374.

Dechent P, Frahm J (2000) Direct mapping of ocular dominance columns in human primary visual cortex. NeuroReport 11:3247-3249.

Dubowitz DJ, Bernheim KA, Chen DY, Bradley Jr WG, Andersen RA (2001) Enhancing fMRI contrast in awake-behaving primates using intravascular magnetite dextran nanopartieles. NeuroReport 12:2335-2340.

Duong TQ, Kim DS, Ugurbil K, Kim SG (2000) Spatiotemporal dynamics of the BOLD fMRI signals: toward mapping submillimeter cortical columns using the early negative response. Magn Reson Med 44:231-242.
Duong TQ, Kim DS, Ugurbil K, Kim SG (2001) Localized cerebral blood flow response at submillimeter columnar resolution. Proc Natl Acad Sci USA 98:10904-10909.

Engel SA, Rumelhart DE, Wandell BA, Lee AT, Glover GH, Chichilnisky EJ, Shadlen MN (1994) fMRI of human visual cortex. Nature 369:525.

Engel SA, Glover GH, Wandell BA (1997) Retinotopic organization in human visual cortex and the spatial precision of functional MRI. Cereb Cortex 7:181-192.

Fize D, Vanduffel W, Nelissen K, Denys K, Chef d'Hotel C, Faugeras O, Orban GA (2003) The retinotopic organization of primate dorsal V4 and surrounding areas: a functional magnetic resonance imaging study in awake monkeys. J Neurosci 23:7395-7406.

Frostig RD, Lieke EE, Ts'o DY, Grinvald A (1990) Cortical functional architecture and local coupling between neuronal activity and the microcirculation revealed by in vivo high-resolution optical imaging of intrinsic signals. Proc Natl Acad Sci USA 87:6082-6086.

Fukuda M, Rajagopalan UM, Homma R, Matsumoto M, Nishizaki M, Tanifuji M (2005) Localization of activity-dependent changes in blood volume to submillimeter-scale functional domains in cat visual cortex. Cereb Cortex 15:823-833.

Fukuda M, Wang P, Moon CH, Tanifuji M, Kim SG (2006) Spatial specificity of the enhanced dip inherently induced by prolonged oxygen consumption in cat visual cortex: implication for columnar resolution functional MRI. NeuroImage 30:70-87.

Goodyear BG, Menon RS (2001) Brief visual stimulation allows mapping of ocular dominance in visual cortex using fMRI. Hum Brain Mapp 14:210-217.

Grinvald A, Lieke E, Frostig RD, Gilbert CD, Wiesel TN (1986) Functional architecture of cortex revealed by optical imaging of intrinsic signals. Nature 324:361-364.

Heeger DJ, Huk AC, Geisler WS, Albrecht DG (2000) Spikes versus BOLD: what does neuroimaging tell us about neuronal activity? Nat Neurosci 3:631-633.

Howarth C, Peppiatt CM, Mobbs P, Attwell D (2005) Pericytes can regulate capillary diameter in rat retina and cerebellum. Soc Neurosci Abstr 31:407.9.

Hudetz AG (1997) Blood flow in the cerebral capillary network: a review emphasizing observations with intravital microscopy. Microcirculation 4:233-252.

Kalatsky VA, Stryker MP (2003) New paradigm for optical imaging: temporally encoded maps of intrinsic signal. Neuron 38:529-545.

Kennan RP, Scanley BE, Innis RB, Gore JC (1998) Physiological basis for BOLD MR signal changes due to neuronal stimulation: separation of blood volume and magnetic susceptibility effects. Magn Reson Med 40:840-846.

Kennerley AJ, Berwick J, Martindale J, Johnston D, Papadakis N, Mayhew JE (2005) Concurrent fMRI and optical measures for the investigation of the hemodynamic response function. Magn Reson Med 54:354-365.

Kim DS, Duong TQ, Kim SG (2000) High-resolution mapping of isoorientation columns by fMRI. Nat Neurosci 3:164-169.

Kim SG, Ugurbil K (2003) High-resolution functional magnetic resonance imaging of the animal brain. Methods 30:28-41.

Leite FP, Tsao D, Vanduffel W, Fize D, Sasaki Y, Wald LL, Dale AM, Kwong KK, Orban GA, Rosen BR, Tootell RB, Mandeville JB (2002) Repeated fMRI using iron oxide contrast agent in awake, behaving macaques at 3 Tesla. NeuroImage 16:283-294.

Logothetis NK, Pauls J, Augath M, Trinath T, Oeltermann A (2001) Neurophysiological investigation of the basis of the fMRI signal. Nature 412:150-157.

Lowel S, Freeman B, Singer W (1987) Topographic organization of the orientation column system in large flat-mounts of the cat visual cortex: a 2-deoxyglucose study. J Comp Neurol 255:401-415.

Lu H, Golay X, Pekar JJ, Van Zijl PC (2003) Functional magnetic resonance imaging based on changes in vascular space occupancy. Magn Reson Med 50:263-274.

Lu H, van Zijl PC, Hendrikse J, Golay X (2004) Multiple acquisitions with global inversion cycling (MAGIC): a multislice technique for vascularspace-occupancy dependent fMRI. Magn Reson Med 51:9-15.

Maldonado PE, Godecke I, Gray CM, Bonhoeffer T (1997) Orientation selectivity in pinwheel centers in cat striate cortex. Science 276:1551-1555. Malonek D, Grinvald A (1996) Interactions between electrical activity and 
cortical microcirculation revealed by imaging spectroscopy: implications for functional brain mapping. Science 272:551-554.

Malonek D, Dirnagl U, Lindauer U, Yamada K, Kanno I, Grinvald A (1997) Vascular imprints of neuronal activity: relationships between the dynamics of cortical blood flow, oxygenation, and volume changes following sensory stimulation. Proc Natl Acad Sci USA 94:14826-14831.

Mandeville JB, Marota JJ (1999) Vascular filters of functional MRI: spatial localization using BOLD and CBV contrast. Magn Reson Med 42:591-598.

Mandeville JB, Marota JJ, Kosofsky BE, Keltner JR, Weissleder R, Rosen BR, Weisskoff RM (1998) Dynamic functional imaging of relative cerebral blood volume during rat forepaw stimulation. Magn Reson Med 39:615-624.

Mandeville JB, Jenkins BG, Chen YC, Choi JK, Kim YR, Belen D, Liu C, Kosofsky BE, Marota JJ (2004) Exogenous contrast agent improves sensitivity of gradient-echo functional magnetic resonance imaging at $9.4 \mathrm{~T}$. Magn Reson Med 52:1272-1281.

Mayhew JE, Askew S, Zheng Y, Porrill J, Westby GW, Redgrave P, Rector DM, Harper RM (1996) Cerebral vasomotion: a $0.1-\mathrm{Hz}$ oscillation in reflected light imaging of neural activity. NeuroImage 4:183-193.

Menon RS, Goodyear BG (1999) Submillimeter functional localization in human striate cortex using BOLD contrast at 4 Tesla: implications for the vascular point-spread function. Magn Reson Med 41:230-235.

Menon RS, Ogawa S, Strupp JP, Ugurbil K (1997) Ocular dominance in human V1 demonstrated by functional magnetic resonance imaging. J Neurophysiol 77:2780-2787.

Moon CH, Fukuda M, Kim SG (2004) Analysis of continuous intrinsic optical imaging data. Soc Neurosci Abstr 30:920.13.

Movshon JA, Thompson ID, Tolhurst DJ (1978) Spatial and temporal contrast sensitivity of neurones in areas 17 and 18 of the cat's visual cortex. J Physiol (Lond) 283:101-120.

Nagaoka T, Zhao F, Wang P, Harel N, Kennan RP, Ogawa S, Kim SG (2006) Increases in oxygen consumption without cerebral blood volume change during visual stimulation under hypotension condition. J Cereb Blood Flow Metab 26:1043-1051.

Narayan SM, Esfahani P, Blood AJ, Sikkens L, Toga AW (1995) Functional increases in cerebral blood volume over somatosensory cortex. J Cereb Blood Flow Metab 15:754-765.

Obrig H, Neufang M, Wenzel R, Kohl M, Steinbrink J, Einhaupl K, Villringer A (2000) Spontaneous low frequency oscillations of cerebral hemodynamics and metabolism in human adults. NeuroImage 12:623-639.

Park SH, Kim SG (2005) MR venography using BOLD contrast at 9.4T. Proc Intl Soc Mag Reson Med 13:1718.

Rao SC, Toth LJ, Sur M (1997) Optically imaged maps of orientation preference in primary visual cortex of cats and ferrets. J Comp Neurol 387:358-370.

Ress D, Backus BT, Heeger DJ (2000) Activity in primary visual cortex predicts performance in a visual detection task. Nat Neurosci 3:940-945.

Sereno MI, Dale AM, Reppas JB, Kwong KK, Belliveau JW, Brady TJ, Rosen BR, Tootell RB (1995) Borders of multiple visual areas in humans revealed by functional magnetic resonance imaging. Science 268:889-893.

Sheth SA, Nemoto M, Guiou M, Walker M, Pouratian N, Hageman N, Toga AW (2004) Columnar specificity of microvascular oxygenation and volume responses: implications for functional brain mapping. J Neurosci 24:634-641.

Shmuel A, Grinvald A (1996) Functional organization for direction of mo- tion and its relationship to orientation maps in cat area 18. J Neurosci 16:6945-6964.

Suh M, Bahar S, Mehta AD, Schwartz TH (2005) Temporal dependence in uncoupling of blood volume and oxygenation during interictal epileptiform events in rat neocortex. J Neurosci 25:68-77.

Takano T, Tian GF, Peng W, Lou N, Libionka W, Han X, Nedergaard M (2006) Astrocyte-mediated control of cerebral blood flow. Nat Neurosci 9:260-267.

Thompson JK, Peterson MR, Freeman RD (2003) Single-neuron activity and tissue oxygenation in the cerebral cortex. Science 299:1070-1072.

Tootell RB, Hadjikhani NK, Vanduffel W, Liu AK, Mendola JD, Sereno MI, Dale AM (1998) Functional analysis of primary visual cortex (V1) in humans. Proc Natl Acad Sci USA 95:811-817.

Tsao DY, Freiwald WA, Tootell RB, Livingstone MS (2006) A cortical region consisting entirely of face-selective cells. Science 311:670-674.

van Bruggen N, Busch E, Palmer JT, Williams SP, de Crespigny AJ (1998) High-resolution functional magnetic resonance imaging of the rat brain: mapping changes in cerebral blood volume using iron oxide contrast media. J Cereb Blood Flow Metab 18:1178-1183.

Vanduffel W, Fize D, Mandeville JB, Nelissen K, Van Hecke P, Rosen BR, Tootell RB, Orban GA (2001) Visual motion processing investigated using contrast agent-enhanced fMRI in awake behaving monkeys. Neuron 32:565-577.

Vanzetta I, Slovin H, Omer DB, Grinvald A (2004) Columnar resolution of blood volume and oximetry functional maps in the behaving monkey; implications for fMRI. Neuron 42:843-854.

Vanzetta I, Hildesheim R, Grinvald A (2005) Compartment-resolved imaging of activity-dependent dynamics of cortical blood volume and oximetry. J Neurosci 25:2233-2244.

Villringer A, Them A, Lindauer U, Einhaupl K, Dirnagl U (1994) Capillary perfusion of the rat brain cortex. An in vivo confocal microscopy study. Circ Res 75:55-62.

Warnking J, Dojat M, Guerin-Dugue A, Delon-Martin C, Olympieff S, Richard N, Chehikian A, Segebarth C (2002) fMRI retinotopic mappingstep by step. NeuroImage 17:1665-1683.

Wei L, Otsuka T, Acuff V, Bereczki D, Pettigrew K, Patlak C, Fenstermacher J (1993) The velocities of red cell and plasma flows through parenchymal microvessels of rat brain are decreased by pentobarbital. J Cereb Blood Flow Metab 13:487-497.

Womelsdorf T, Eysel UT, Kisvarday ZF (2001) Comparison of orientation maps obtained with different number of stimulus orientations. NeuroImage 13:1131-1139.

Zepeda A, Arias C, Sengpiel F (2004) Optical imaging of intrinsic signals: recent developments in the methodology and its applications. J Neurosci Methods 136:1-21.

Zhao F, Wang P, Hendrich K, Kim SG (2005) Spatial specificity of cerebral blood volume-weighted fMRI responses at columnar resolution. NeuroImage 27:416-424.

Zhao F, Wang P, Hendrich K, Ugurbil K, Kim SG (2006) Cortical layerdependent BOLD and CBV responses measured by spin-echo and gradient-echo fMRI: insights into hemodynamic regulation. NeuroImage 30:1149-1160.

Zonta M, Angulo MC, Gobbo S, Rosengarten B, Hossmann KA, Pozzan T, Carmignoto G (2003) Neuron-to-astrocyte signaling is central to the dynamic control of brain microcirculation. Nat Neurosci 6:43-50. 